\title{
Electric dipole moment of the tau lepton revisited
}

\author{
Werner Bernreuther ${ }^{1,{ }^{*}}$ Long Chen, ${ }^{1, \dagger}$ and Otto Nachtmann ${ }^{2, *}$ \\ ${ }^{1}$ Institut für Theoretische Teilchenphysik und Kosmologie, RWTH Aachen University, \\ 52056 Aachen, Germany \\ ${ }^{2}$ Institut für Theoretische Physik, Universität Heidelberg, 69120 Heidelberg, Germany
}

(Received 27 January 2021; accepted 12 April 2021; published 14 May 2021)

\begin{abstract}
We reconsider the issue of the search for a nonzero electric dipole form factor (EDM) $d_{\tau}(s)$ using optimal observables in $\tau^{+} \tau^{-}$production by $e^{+} e^{-}$collisions in the center-of-mass energy range from the $\tau$-pair threshold to about $\sqrt{s} \sim 15 \mathrm{GeV}$. We discuss the general formalism of optimal observables and apply it to two $C P$-odd observables that are sensitive to the real and imaginary part of $d_{\tau}(s)$, respectively. We compute the expectation values and covariances of these optimal $C P$ observables for $\tau$-pair production at $\sqrt{s}=10.58 \mathrm{GeV}$ with subsequent decays of $\tau^{ \pm}$into major leptonic or semihadronic modes. For the $\tau$ decays to two pions and three charged pions we take the full kinematic information of the hadronic system into account. Assuming that the Belle II experiment at the KEKB accelerator will eventually analyze data corresponding to an integrated luminosity of $50 \mathrm{ab}^{-1}$ and applying acceptance cuts on the final-state pions, we find that 1 s.d. sensitivities $\delta \operatorname{Re} d_{\tau}=6.8 \times 10^{-20} e \mathrm{~cm}$ and $\delta \operatorname{Im} d_{\tau}=4.0 \times 10^{-20} e \mathrm{~cm}$ can be obtained with events where both $\tau$ 's decay semihadronically. We consider also the ideal case that no cuts on the finalstate particles are applied. With $50 \mathrm{ab}^{-1}$ at $\sqrt{s}=10.58 \mathrm{GeV}$ corresponding to $4.5 \times 10^{10} \tau^{+} \tau^{-}$events we find the 1 s.d. sensitivities $\delta \operatorname{Re} d_{\tau}=5.8 \times 10^{-20} e \mathrm{~cm}$ and $\delta \operatorname{Im} d_{\tau}=3.2 \times 10^{-20} e \mathrm{~cm}$, again for events where both $\tau$ leptons decay semihadronically. Furthermore, we analyze the potential magnitude of the $\tau$ EDM form factor in the type-II two-Higgs doublet extension and in two scalar leptoquark extensions of the Standard Model, taking into account phenomenological constraints.
\end{abstract}

DOI: 10.1103/PhysRevD.103.096011

\section{INTRODUCTION}

The search for electric dipole moments (EDMs) of fundamental fermions is an important aspect of experimental investigations hunting for physics beyond the Standard Model (SM) of particle physics, in particular for $C P$ violation beyond the Kobayashi-Maskawa mechanism. So far only upper bounds for these EDMs exist [1]. For the electron an impressive upper limit was obtained rather recently by the ACME Collaboration [2]. The best muon EDM limit to date was set by the Muon $(g-2)$ Collaboration [3]. These limits are

$$
\left|d_{e}\right|<1.1 \times 10^{-29} e \mathrm{~cm} \quad \text { at } 90 \% \text { C.L. }
$$

\footnotetext{
*breuther@physik.rwth-aachen.de

†ongchen@physik.rwth-aachen.de

o.nachtmann@thphys.uni-heidelberg.de
}

Published by the American Physical Society under the terms of the Creative Commons Attribution 4.0 International license. Further distribution of this work must maintain attribution to the author(s) and the published article's title, journal citation, and DOI. Funded by SCOAP ${ }^{3}$.

$$
\left|d_{\mu}\right|<1.8 \times 10^{-19} e \mathrm{~cm} \quad \text { at } 95 \% \text { C.L. }
$$

The lifetime of the $\tau$ lepton is too short to allow for the measurement of its static moments. Instead, information on the nonstatic $\tau$ EDM form factor ${ }^{1}$ can be retrieved, for instance, from the measurement of $C P$-violating correlations in $\tau$-pair production by $e^{+} e^{-}$collisions. The $\tau$ EDM form factor can be a complex quantity for timelike momentum transfer. The best limits to date on its real and imaginary parts were obtained by the Belle I Collaboration [4] at $q^{2}=(10.58 \mathrm{GeV})^{2}$ :

$$
\begin{aligned}
-2.2 \times 10^{-17} e \mathrm{~cm} & <\operatorname{Re} d_{\tau}\left(q^{2}\right) \\
& <4.5 \times 10^{-17} e \mathrm{~cm} \text { at } 95 \% \text { C.L. } \\
-2.5 \times 10^{-17} e \mathrm{~cm} & <\operatorname{Im} d_{\tau}\left(q^{2}\right) \\
& <0.8 \times 10^{-17} e \mathrm{~cm} \text { at 95\%C.L. }
\end{aligned}
$$

In a series of articles where two of the authors of this paper were involved, ways of searching for $C P$-violating effects in $e^{+} e^{-}$collisions, in particular for a nonzero $\tau$

\footnotetext{
${ }^{1}$ In this paper we use the acronym EDM for both the static moment and the form factor at $q^{2} \neq 0$.
} 
EDM, were proposed [5-9]. The observables and results of $[6,9]$ were used in the experimental searches for an EDM form factor of the $\tau$ lepton by [4] and earlier by the ARGUS Collaboration [10] that obtained the results

$$
\begin{aligned}
& \left|\operatorname{Re} d_{\tau}\left(q^{2}\right)\right|<4.6 \times 10^{-16} e \mathrm{~cm} \quad \text { at } 95 \% \text { C.L. } \\
& \left|\operatorname{Im} d_{\tau}\left(q^{2}\right)\right|<1.8 \times 10^{-16} e \mathrm{~cm} \quad \text { at } 95 \% \text { C.L. }
\end{aligned}
$$

at a c.m. energy $\sqrt{s}=\sqrt{q^{2}}=10 \mathrm{GeV}$ of the reaction $e^{+} e^{-} \rightarrow \tau^{+} \tau^{-}$. For reviews of the search results for the $\tau$ EDM and its weak dipole form factor (the analogue of the EDM for the coupling of the $Z$ boson to fermions); see, for instance, $[11,12]$. Further discussions of possible measurements of the anomalous magnetic moment and the EDM of the $\tau$ lepton can be found in [13-17] and references therein.

The experimentation at Belle II [18] which started recently at the KEKB accelerator offers new possibilities for measuring the $\tau$ EDM form factor, in particular, because a huge number of recorded $\tau$-pair events are expected at the end of data taking [19]. Also the BES III experiment, where $e^{+} e^{-}$collisions at a center-of-mass (c.m.) energy $\sqrt{s} \sim$ $4 \mathrm{GeV}$ are studied, expects to collect and analyze a large number of $\tau^{+} \tau^{-}$pairs [20]. Therefore, we reconsider the issue with particular emphasis on using optimal observables [21-23] for tracing the $\tau$ EDM form factor in $\tau$-pair production at c.m. energies from threshold up to about $15 \mathrm{GeV}$ where the contribution from $Z$-boson exchange is negligible. In our numerical analysis we consider $\tau$-pair production at $\sqrt{s}=10.58 \mathrm{GeV}$. Moreover, we analyze this form factor in a few SM extensions that can induce a potentially sizable $\tau$ EDM [24].

Our paper is organized as follows. In Sec. II we recall the form factor decomposition of the $\gamma \tau \tau$ vertex and in particular the definition of the $\tau$ EDM form factor. In Sec. III we discuss the production and decay matrices for the process $e^{+} e^{-} \rightarrow \tau^{+} \tau^{-}$with the $\tau$ 's decaying into one, two, or three particles that are measured in an experiment. Section IV deals with simple and optimal observables [21-23] for tracing the EDM of the $\tau$ lepton. Section V contains our numerical results, in particular our estimates of the sensitivities with which the real and the imaginary parts of the $\tau$ EDM form factor can be measured in various $\tau$ decay channels. In Sec. VI we consider the $\tau$ EDM form factor in a type-II two-Higgs doublet extension and in two leptoquark extensions of the SM and analyze the potential magnitude of the $\tau$ EDM taking into account experimental constraints. Moreover, we show that within these models $C P$-violating box contributions to the $S$-matrix element of $e^{+} e^{-} \rightarrow \tau^{+} \tau^{-}$are negligible as compared to that of the $\tau$ EDM form factor. We conclude in Sec. VII. In the Appendix A we list the density matrices for several major decays of polarized $\tau^{ \pm}$leptons. In particular, we present the explicit form of the differential decay density matrices for $\tau \rightarrow 2 \pi \nu_{\tau}$ and $\tau \rightarrow 3 \pi \nu_{\tau}$. Appendix B contains a detailed analysis of the expectation values and covariances of the $C P$-odd optimal observables used in Sec. $\mathrm{V}$ in various $\tau^{+} \tau^{-}$ decay channels.

\section{FORM FACTORS}

We consider $\tau^{+} \tau^{-}$production in $e^{+} e^{-}$collisions at c.m. energies $\sqrt{s}$ from threshold up to about $15 \mathrm{GeV}$, with $\tau^{-}$ and $\tau^{+}$decaying into a final state $A$ and $\bar{B}$, respectively,

$e^{+}\left(p_{+}\right)+e^{-}\left(p_{-}\right) \rightarrow \tau^{+}\left(k_{+}, \alpha\right)+\tau^{-}\left(k_{-}, \beta\right) \rightarrow \bar{B}+A$.

The four-momenta and the corresponding three-momenta are denoted in the $e^{+} e^{-}$c.m. frame by $p_{ \pm}=\left(p_{ \pm}^{0}, \mathbf{p}_{ \pm}\right)^{T}$, $k_{ \pm}=\left(k_{ \pm}^{0}, \mathbf{k}_{ \pm}\right)^{T}$. We consider unpolarized electrons and positrons and neglect their masses; the labels $\alpha, \beta \in$ $\{ \pm 1 / 2\}$ denote the spin indices of the tau leptons. In the c.m. frame we have $\mathbf{p}_{+}+\mathbf{p}_{-}=\mathbf{k}_{+}+\mathbf{k}_{-}=0$.

For unpolarized $e^{+}$and $e^{-}$the initial state is described by a $C P$-invariant density matrix. Thus, any nonzero $C P$-odd correlation observed in the final state indicates a genuine $C P$-violating effect that can be located in the production and/or in the decays of the $\tau$ 's. We consider tau-pair production by one-photon-exchange only. At the energies considered here $Z$-boson exchange is negligible. This will be justified at the end of this section. The diagram shown in Fig. 1 exhibits this approximation with the full photon propagator

$$
i \Delta_{\mu \nu}^{(\gamma)}(q)=\frac{-i g_{\mu \nu}}{q^{2}\left[1+e^{2} \Pi_{c}\left(q^{2}\right)\right]},
$$

where $\Pi_{c}\left(q^{2}\right)$ is the vacuum-polarization function; see, e.g., Eq. (19.45) of [25]. For instance, at the mass of the $\Upsilon(4 \mathrm{~S})$ resonance, at $\sqrt{q^{2}}=10.58 \mathrm{GeV}$, this vacuum polarization effect produces an enhancement of the cross section. For a detailed discussion of the $\tau$-pair cross section at this energy, including radiative corrections, we refer to [26]. Below we consider only normalized expectation values of $C P$ observables where such resonance enhancements enter only through the number of events which we take as input from experiment.

In the following we assume that the only source of $C P$ violation in the diagram of Fig. 1 is due to a nonzero EDM form factor in the $\gamma \tau \tau$ vertex. This vertex is given by the following one-particle irreducible (1PI) matrix element of the electromagnetic current $J_{\lambda}^{\mathrm{em}}$ between the vacuum and the $\tau^{+} \tau^{-}$final state:

$$
\begin{aligned}
& \left\langle\tau^{-}\left(k_{-}, \beta\right), \tau^{+}\left(k_{+}, \alpha\right) \operatorname{out}\left|J_{\lambda}^{\mathrm{em}}(0)\right| 0\right\rangle \\
& =-\bar{u}_{\beta}\left(k_{-}\right)\left[e F_{1}\left(q^{2}\right) \gamma_{\lambda}+\frac{i}{2 m_{\tau}} \sigma_{\lambda \mu} q^{\mu} e F_{2}\left(q^{2}\right)+d_{\tau}\left(q^{2}\right) \sigma_{\lambda \mu} q^{\mu} \gamma_{5}\right. \\
& \left.+\frac{1}{8 \pi} A\left(q^{2}\right)\left(q^{2} \gamma_{\lambda}-2 m_{\tau} q_{\lambda}\right) \gamma_{5}\right] v_{\alpha}\left(k_{+}\right)
\end{aligned}
$$




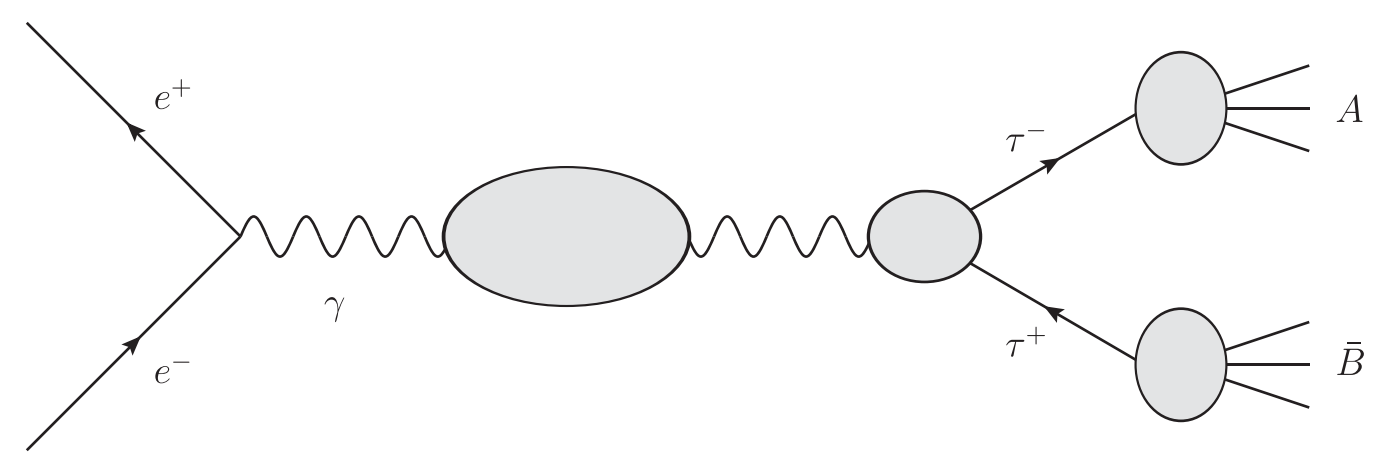

FIG. 1. The reaction (5) in the one-photon-exchange approximation.

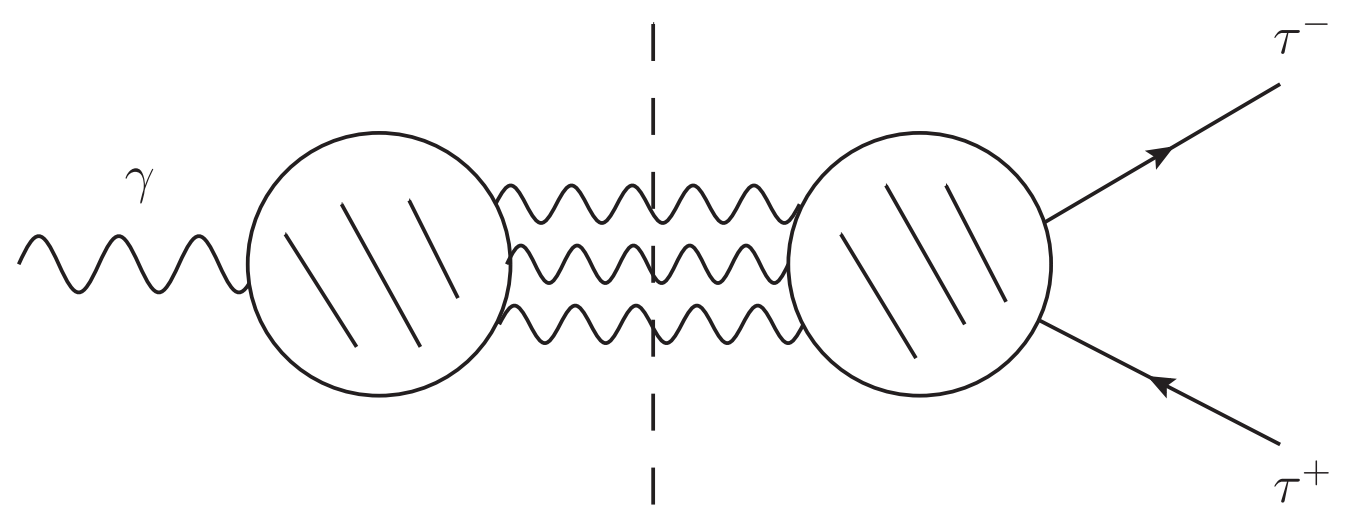

FIG. 2. A cut diagram leading to an imaginary part of the form factors in (7) for $q^{2}>0$.

where $q=k_{+}+k_{-}$. The right-hand side of (7) represents the most general decomposition of this matrix element taking into account the conservation of the electromagnetic current. Moreover, $e=\sqrt{4 \pi \alpha_{\mathrm{em}}}>0$ denotes the $\tau^{+}$ charge, and we use the $\gamma$-matrix conventions of [25]. Note that the order of $\tau^{-}$and $\tau^{+}$in the matrix element (7) matters because we are dealing with fermions. The form factors $F_{1,2}\left(q^{2}\right), d_{\tau}\left(q^{2}\right)$, and $A\left(q^{2}\right)$ are analytic functions of $q^{2}$ in the complex $q^{2}$ plane with a cut on the positive real axis satisfying

$$
\begin{aligned}
F_{i}\left(q^{2 *}\right)^{*} & =F_{i}\left(q^{2}\right), \quad i=1,2 \\
d_{\tau}\left(q^{2 *}\right)^{*} & =d_{\tau}\left(q^{2}\right) \\
A\left(q^{2 *}\right)^{*} & =A\left(q^{2}\right)
\end{aligned}
$$

That is, on the real $q^{2}$ axis, the form factors are real functions for $q^{2}<0$ and can have imaginary parts for $q^{2}>0$. At higher order in $\alpha_{\text {em }}$ these cuts start at $q^{2}=0$ due to cut diagrams of the type shown in Fig. 2 with three photons in the intermediate state. In the decomposition (7) we have $q^{2} \geq 4 m_{\tau}^{2}$, and we have to set $q^{2}+i \varepsilon$, that is, to take $q^{2}$ above the cut.

Next we recall the transformation properties of the $\gamma \tau \tau$ coupling terms associated with the four form factors in (7) under charge conjugation $(C)$, parity $(P)$, and $C P$.
Assuming that the interaction is invariant under these transformations and using the transformation of $J_{\lambda}^{\mathrm{em}}(x)$ under $C, P$, and $C P$, one gets the transformation properties listed in Table I.

The $e^{+} e^{-} \rightarrow \tau^{+} \tau^{-}$amplitude can receive also $C P$-odd 1PI box contributions, for instance, contributions with Lorentz structure $(\bar{e} e)\left(\bar{\tau} i \gamma_{5} \tau\right)$. We do not take such contributions into account in the following. We discuss a few SM extensions in Sec. VI that can induce sizable $\tau$ EDM form factors. For these models we show in Sec. VIC that the $C P$-violating box contributions can be neglected as compared to that of the induced $\tau$ EDM form factor.

For the matrix elements of the current between $\tau^{-}$and $\tau^{+}$ states, respectively, we get, using the standard crossing relations:

TABLE I. Transformation properties of the $\gamma \tau \tau$ coupling terms corresponding to the four form factors in the decomposition of the matrix element (7) of the electromagnetic current.

\begin{tabular}{llll}
\hline \hline & $C$ & $P$ & $C P$ \\
\hline$F_{1}\left(q^{2}\right)$ & + & + & + \\
$F_{2}\left(q^{2}\right)$ & + & + & + \\
$d_{\tau}\left(q^{2}\right)$ & + & - & - \\
$A\left(q^{2}\right)$ & - & - & + \\
\hline \hline
\end{tabular}




$$
\begin{aligned}
& \left\langle\tau^{-}\left(k^{\prime}, \beta^{\prime}\right)\left|J_{\lambda}^{\mathrm{em}}(0)\right| \tau^{-}(k, \beta)\right\rangle=-\bar{u}_{\beta^{\prime}}\left(k^{\prime}\right) \Gamma_{\lambda}(q) u_{\beta}(k), \\
& \left\langle\tau^{+}\left(k^{\prime}, \alpha^{\prime}\right)\left|J_{\lambda}^{\mathrm{em}}(0)\right| \tau^{+}(k, \alpha)\right\rangle=\bar{v}_{\alpha}(k) \Gamma_{\lambda}(q) v_{\alpha^{\prime}}\left(k^{\prime}\right),
\end{aligned}
$$

where the vertex function $\Gamma_{\lambda}(q)$ is given by the expression in the square brackets of Eq. (7) with $q=k^{\prime}-k$ and $q^{2} \leq 0$.

The form factor $F_{1}\left(q^{2}\right)$ is the electric or Dirac form factor with the normalization

$$
F_{1}(0)=1
$$

The magnetic or Pauli form factor $F_{2}\left(q^{2}\right)$ at $q^{2}=0$ yields the $\tau$ anomalous magnetic moment:

$$
F_{2}(0)=a_{\tau}=\frac{1}{2}\left(g_{\tau}-2\right)
$$

The $\tau^{-}$and $\tau^{+}$electric dipole moments, respectively, are obtained from the EDM form factor $d_{\tau}\left(q^{2}\right)$ at $q^{2}=0$ :

$$
d_{\tau^{-}}=-d_{\tau^{+}}=d_{\tau}(0)
$$

The form factor $A\left(q^{2}\right)$ at $q^{2}=0$ defines the anapole moment [27-30] of the $\tau^{-}$:

$$
A_{\tau^{-}}=A(0)
$$

For a $\tau^{-}$at rest, $k=k_{R}=\left(m_{\tau}, \mathbf{0}\right)^{T}$, one has

$$
\left\langle\left.\tau^{-}\left(k_{R}, \beta^{\prime}\right)\left|(-\pi) \int d^{3} x\right| \boldsymbol{x}\right|^{2} \boldsymbol{J}^{\mathrm{em}}(\boldsymbol{x}, 0) \mid \tau^{-}\left(k_{R}, \beta\right)\right\rangle=\frac{1}{2} \boldsymbol{\sigma}_{\beta^{\prime} \beta} A_{\tau^{-}} .
$$

A comment on the gauge invariance of the form-factor decomposition of the vertex function (7) is in order. Electromagnetic gauge invariance is obvious, because conservation of the electromagnetic current was used in the decomposition of (7). As to the invariance with respect to the electroweak gauge group $\mathrm{SU}(2) \times \mathrm{U}(1)$ : The static moments at $q^{2}=0$, in particular the anomalous magnetic and electric dipole moment and the anapole moment, are gauge invariant, as they correspond to terms in the $\tau \rightarrow \tau S$-matrix element in the soft-photon limit. Yet, for obtaining a gauge-invariant amplitude for $e^{+} e^{-} \rightarrow \tau^{+} \tau^{-}$one cannot, of course, use (7) in isolation, but must take into account all contributions (including box contributions at one-loop order and beyond) to the $S$-matrix element order by order in the electroweak couplings. However, in the following we use only the treelevel $\gamma \tau \tau$ vertex supplemented by the $\tau$ EDM form factor. The $\tau$ EDM is extremely small in the SM, as will be briefly reviewed at the beginning of Sec. VI. Thus, a sizable value for $d_{\tau}$ must come from "beyond the Standard Model" (BSM) physics. In Sec. VI we discuss a few BSM extensions that can induce a sizable $\tau$ EDM form factor and compute it at oneloop order. The form factors $d_{\tau}\left(q^{2}\right)$ given in that section are invariant with respect to the electroweak gauge group.

As is well-known one may introduce a $\tau$ EDM, together with an analogous $C P$-violating weak dipole moment $(\mathrm{WDM}) d_{\tau}^{Z}$ in the $Z \tau \tau$ vertex, by using a $\mathrm{SU}(3) \times \mathrm{SU}(2) \times$ $\mathrm{U}(1)$ invariant effective Lagrangian approach for BSM couplings. Imposing baryon and lepton number conservation the leading gauge-invariant operators have mass dimension 6 [31] and the relevant effective Lagrangian takes the form (see, for instance, [32])

$$
\begin{aligned}
\mathcal{L}_{\mathrm{eff}}(x)= & -i \frac{c_{1}}{\Lambda^{2}} \bar{\tau}_{R}(x) \sigma^{\mu \nu} \phi^{\dagger}(x)\left[g^{\prime} \frac{\tau^{a}}{2} W_{\mu \nu}^{a}(x)-\frac{g}{2} B_{\mu \nu}(x)\right] L_{L}(x) \\
& -i \frac{c_{2}}{\Lambda^{2}} \bar{\tau}_{R}(x) \sigma^{\mu \nu} \phi^{\dagger}(x)\left[g \frac{\tau^{a}}{2} W_{\mu \nu}^{a}(x)+\frac{g^{\prime}}{2} B_{\mu \nu}(x)\right] L_{L}(x)+\text { H.c. }
\end{aligned}
$$

Here $c_{1}$ and $c_{2}$ are dimensionless real coupling constants, $\Lambda \gg \mathrm{v}$ denotes the energy scale of new physics that is assumed to be considerably larger than the electroweak symmetry breaking scale $\mathrm{v}=246 \mathrm{GeV}, g$ and $g^{\prime}$ are the $\mathrm{SU}(2)$ and $\mathrm{U}(1)$ gauge couplings, respectively, $W_{\mu \nu}$ and $B_{\mu \nu}$ are the gauge field strength tensors corresponding to these groups, $\phi$ is the Higgs doublet field, and $\tau_{R}$ and $L_{L}^{T}=\left(\nu_{\tau}, \tau\right)_{L}^{T}$ are the right-handed singlet and lefthanded lepton doublet fields of the third generation. (Our notation follows [33].) After spontaneous symmetry breaking the effective Lagrangian (16) contains the EDM interactions

$$
\begin{aligned}
\mathcal{L}_{\text {eff }}(x) \supset & -\frac{i}{2} d_{\tau} \bar{\tau}(x) \sigma^{\mu \nu} \gamma_{5} \tau(x) F_{\mu \nu}(x) \\
& -\frac{i}{2} d_{\tau}^{Z} \bar{\tau}(x) \sigma^{\mu \nu} \gamma_{5} \tau(x) Z_{\mu \nu}(x),
\end{aligned}
$$

where $F_{\mu \nu}=\partial_{\mu} A_{\nu}-\partial_{\nu} A_{\mu}$ and $Z_{\mu \nu}=\partial_{\mu} Z_{\nu}-\partial_{\nu} Z_{\mu}$ are, respectively, the Abelian field strength tensors of the photon and $Z$ boson and $d_{\tau}$ and $d_{\tau}^{Z}$ the electric and weak dipole moments of the $\tau$ lepton:

$$
d_{\tau}=\frac{\mathrm{v}_{0}}{\Lambda^{2}} \frac{\sqrt{g^{2}+g^{\prime 2}}}{\sqrt{2}} c_{1}, \quad d_{\tau}^{Z}=\frac{\mathrm{v}_{0}}{\Lambda^{2}} \frac{\sqrt{g^{2}+g^{\prime 2}}}{\sqrt{2}} c_{2} .
$$


This approach constitutes a possibility to introduce the $\tau$ EDM and WDM in a way that respects electroweak gauge invariance. Using the Hermitian Lagrangian (17) to leading order corresponds to setting

$$
\operatorname{Re} d_{\tau}\left(q^{2}\right)=d_{\tau}, \quad \operatorname{Im} d_{\tau}\left(q^{2}\right)=0,
$$

and likewise for $d_{\tau}^{Z}$. In this framework imaginary parts of $d_{\tau}$ and $d_{\tau}^{Z}$ will be generated by diagrams involving both $\mathcal{L}_{\text {eff }}$ of Eq. (17) and SM couplings at higher order. We shall take into account in the following that $\operatorname{Im} d_{\tau}$ can be nonzero for $q^{2}>0$ but we neglect, as already mentioned above, the contribution from $Z$-boson exchange, in particular the contribution from $d_{\tau}^{Z}$. This can be justified as follows. Equation (18) shows that $d_{\tau}$ and $d_{\tau}^{Z}$ will be of the same order of magnitude if the coupling constants $c_{1}$ and $c_{2}$ are of comparable size. This is the case, for instance, in the BSM models considered in Sec. VI, as was shown in [24]. At energies $\sqrt{s} \ll m_{Z}$ that we consider in this paper, the effects of $d_{\tau}^{Z}$ resulting from $Z$-boson exchange are then negligible compared to those of $d_{\tau}$, as is the contribution resulting from the interference of the SM Z-boson exchange amplitude with the amplitude involving $d_{\tau}$. One can see this explicitly from the formulas given in [9] where both $\gamma$ and Z-boson exchange were taken into account. From Eq. (3.10) of [9] we find that for c.m. energies $\sqrt{s} \approx 10 \mathrm{GeV}$ that we are considering $Z$-boson exchange contributions are suppressed by a factor of order

$$
s / m_{Z}^{2} \approx 10^{-2}
$$

This holds for $\sqrt{s}$ in the continuum and at the $\Upsilon(4 S)$ resonance where the suppression factor (20) is a few percent smaller because there the photon contribution is enhanced as compared to the continuum value; see [26].

\section{MATRIX ELEMENTS, PRODUCTION, AND DECAY MATRICES}

We are interested in analyzing $C P$-violating effects in $\tau$-pair production generated by a nonzero $\tau$ EDM form factor. Therefore we shall analyze the reactions (5) by considering on-shell $\tau$-pair production by one-photon exchange, including the $\tau$ EDM form factor in the $\gamma \tau \tau$ vertex, followed by the decays of $\tau^{-}$and $\tau^{+}$into the final states $A$ and $\bar{B}$, respectively. The $\tau$ spin correlations and polarizations will be taken into account. [The reactions (5) were investigated in [9] for arbitrary c.m. energies for photon and Z-boson exchange including besides the EDM also the weak dipole form factor of the $\tau$ lepton.]

As to the decay channels $A$ and $\bar{B}$, we consider two cases:

(i) Only one charged particle of $A$ and one of $\bar{B}$ are measured:

$$
\tau^{-} \rightarrow a\left(q_{-}\right)+X, \quad \tau^{+} \rightarrow \bar{b}\left(q_{+}\right)+X^{\prime} .
$$

Examples from the main decay modes of $\tau^{-}$are

$$
\tau^{-} \rightarrow e^{-}\left(q_{-}\right) \bar{\nu}_{e} \nu_{\tau}, \quad \mu^{-}\left(q_{-}\right) \bar{\nu}_{\mu} \nu_{\tau}, \quad \pi^{-}\left(q_{-}\right) \nu_{\tau}, \quad \pi^{-}\left(q_{-}\right) \pi^{0} \nu_{\tau}, \quad \pi^{-}\left(q_{-}\right) \pi^{0} \pi^{0} \nu_{\tau}, \quad \pi^{-}\left(q_{-}\right) \pi^{+} \pi^{-} \nu_{\tau},
$$

and the respective charge-conjugate $\tau^{+}$decays. The decay modes (22) include, in particular, $\tau$ decays to one charged prong.

(ii) We shall also treat the case where more than one particle from $\tau$ decay is observed, specifically the decay to two pions via a $\rho$ and $\rho^{\prime}$ meson and the decay to the $a_{1}$ meson, respectively, to three charged pions:

$$
\begin{aligned}
\tau^{-} \rightarrow \pi^{-}\left(q_{1}\right) \pi^{0}\left(q_{2}\right) \nu_{\tau}, & \tau^{+} \rightarrow \pi^{+}\left(\bar{q}_{1}\right) \pi^{0}\left(\bar{q}_{2}\right) \bar{\nu}_{\tau}, \\
\tau^{-} \rightarrow \pi^{-}\left(q_{1}\right) \pi^{-}\left(q_{2}\right) \pi^{+}\left(q_{3}\right) \nu_{\tau}, & \tau^{+} \rightarrow \pi^{+}\left(\bar{q}_{1}\right) \pi^{+}\left(\bar{q}_{2}\right) \pi^{-}\left(\bar{q}_{3}\right) \bar{\nu}_{\tau} .
\end{aligned}
$$

For on-shell $\tau$-pair production and decay the cross section of (5) can be written as a product of the production density matrix $R$ for $e^{+} e^{-} \rightarrow \tau^{+} \tau^{-}$times the density matrices $\mathcal{D}_{\alpha^{\prime} \alpha}^{\bar{B}}$ and $\mathcal{D}_{\beta^{\prime} \beta}^{A}$ that describe the decays of polarized $\tau^{+} \rightarrow \bar{B}$ and $\tau^{-} \rightarrow A$, respectively. The production density matrix $R$ is defined as follows:

$$
\begin{aligned}
R_{\alpha \alpha^{\prime} \beta \beta^{\prime}}= & \frac{1}{4} \sum_{\gamma, \delta}\left\langle\tau^{+}\left(k_{+}, \alpha\right), \tau^{-}\left(k_{-}, \beta\right)|\mathcal{T}| e^{+}\left(p_{+}, \gamma\right), e^{-}\left(p_{-}, \delta\right)\right\rangle \\
& \times\left\langle\tau^{+}\left(k_{+}, \alpha^{\prime}\right), \tau^{-}\left(k_{-}, \beta^{\prime}\right)|\mathcal{T}| e^{+}\left(p_{+}, \gamma\right), e^{-}\left(p_{-}, \delta\right)\right\rangle^{*}
\end{aligned}
$$

where $\gamma, \delta$ are the spin indices of $e^{+}$and $e^{-}$, respectively. For a decay of $\tau^{-}$according to case (i) above the corresponding decay density matrix is given by 


$$
\begin{aligned}
\mathcal{D}_{\beta^{\prime} \beta}^{a}\left(\tau^{-}\left(k_{-}\right) \rightarrow a\left(q_{-}\right)+X\right)= & \Gamma^{-1}\left(\tau^{-} \rightarrow A\right) \frac{1}{2 m_{\tau}} \int d \Gamma_{X}(2 \pi)^{4} \delta^{(4)}\left(k_{-}-q_{-}-q_{X}\right) \\
& \times\left\langle a\left(q_{-}\right), X|\mathcal{T}| \tau^{-}\left(k_{-}, \beta\right)\right\rangle\left\langle a\left(q_{-}\right), X|\mathcal{T}| \tau^{-}\left(k_{-}, \beta^{\prime}\right)\right\rangle^{*}
\end{aligned}
$$

Here the normalization is chosen such that

$$
\int \frac{d^{3} q_{-}}{(2 \pi)^{3} 2 q_{-}^{0}} \mathcal{D}_{\beta^{\prime} \beta}^{a}\left(\tau^{-}\left(k_{-}\right) \rightarrow a\left(q_{-}\right)+X\right)=\delta_{\beta^{\prime} \beta}\left\langle n_{a}\right\rangle_{A},
$$

where $\left\langle n_{a}\right\rangle_{A}$ is the mean multiplicity of particle $a$ in channel $A$. Formulas analogous to (26) and (27) apply if decays $\tau^{+} \rightarrow \bar{b}+X^{\prime}$ according to case (i) are considered.

Thus the cross section for the two-particle inclusive reactions

$$
e^{+} e^{-} \rightarrow \tau^{+} \tau^{-} \rightarrow \bar{B}+A
$$

where

$$
A=a\left(q_{-}\right)+X, \quad \bar{B}=\bar{b}\left(q_{+}\right)+X^{\prime},
$$

is given in the narrow-width approximation of the intermediate $\tau$ leptons by

$$
\begin{aligned}
d \sigma_{a \bar{b}}= & \frac{\sqrt{1-4 m_{\tau}^{2} / s}}{16 \pi s} \frac{d \Omega_{k_{+}}}{4 \pi} \operatorname{Br}\left(\tau^{-} \rightarrow A\right) \operatorname{Br}\left(\tau^{+} \rightarrow \bar{B}\right) \\
& \times R_{\alpha \alpha^{\prime} \beta \beta^{\prime}} \frac{d^{3} q_{-}}{(2 \pi)^{3} 2 q_{-}^{0}} \mathcal{D}_{\beta^{\prime} \beta}^{a}\left[\tau^{-} \rightarrow a\left(q_{-}\right)+X\right] \frac{d^{3} q_{+}}{(2 \pi)^{3} 2 q_{+}^{0}} \mathcal{D}_{\alpha^{\prime} \alpha}^{\bar{b}}\left[\tau^{+} \rightarrow \bar{b}\left(q_{+}\right)+X^{\prime}\right],
\end{aligned}
$$

where $s=\left(p_{+}+p_{-}\right)^{2}$, the solid angle element $d \Omega_{k_{+}}$corresponds to the momentum vector $\mathbf{k}_{+}$in the $e^{+} e^{-}$c.m. frame, and $\operatorname{Br}\left(\tau^{-} \rightarrow A\right)$ and $\operatorname{Br}\left(\tau^{+} \rightarrow \bar{B}\right)$ denote the branching fractions for the decays $\tau^{-} \rightarrow A$ and $\tau^{+} \rightarrow \bar{B}$, respectively. ${ }^{2}$

For $\tau$ decay to three charged pions whose four-momenta are all measured in an experiment, we define the corresponding decay density matrix by

$$
\begin{aligned}
& \mathcal{D}_{\beta^{\prime} \beta}^{A}\left(\tau^{-}\left(k_{-}\right) \rightarrow \pi^{-}\left(q_{1}\right) \pi^{-}\left(q_{2}\right) \pi^{+}\left(q_{3}\right) \nu_{\tau}\right) \\
&= \Gamma^{-1}\left(\tau^{-} \rightarrow \pi^{-} \pi^{-} \pi^{+} \nu_{\tau}\right) \frac{1}{2 m_{\tau}} \int \frac{d^{3} q_{4}}{(2 \pi)^{3} 2 q_{4}^{0}}(2 \pi)^{4} \delta^{(4)}\left(k_{-}-q_{1}-q_{2}-q_{3}-q_{4}\right) \\
& \times\left\langle\pi^{-}\left(q_{1}\right) \pi^{-}\left(q_{2}\right) \pi^{+}\left(q_{3}\right) \nu_{\tau}|\mathcal{T}| \tau^{-}\left(k_{-}, \beta\right)\right\rangle\left\langle\pi^{-}\left(q_{1}\right) \pi^{-}\left(q_{2}\right) \pi^{+}\left(q_{3}\right) \nu_{\tau}|\mathcal{T}| \tau^{-}\left(k_{-}, \beta^{\prime}\right)\right\rangle^{*},
\end{aligned}
$$

where $q_{4}$ is the four-momentum of $\nu_{\tau}$, and analogously for the decay $\tau^{+} \rightarrow \pi^{+} \pi^{+} \pi^{-} \bar{\nu}_{\tau}$. The normalization is

$$
\int \prod_{i=1}^{3} \frac{d^{3} q_{i}}{(2 \pi)^{3} 2 q_{i}^{0}} \mathcal{D}_{\beta^{\prime} \beta}^{A}\left(\tau^{-}\left(k_{-}\right) \rightarrow \pi^{-}\left(q_{1}\right) \pi^{-}\left(q_{2}\right) \pi^{+}\left(q_{3}\right) \nu_{\tau}\right)=2 \delta_{\beta^{\prime} \beta},
$$

corresponding to the $\pi^{-}$multiplicity 2 in this channel. If the analysis is restricted to three pions in a suitably defined invariant mass region around the nominal $a_{1}$ mass one has to take into account the corresponding phase-space cuts in $\Gamma\left(\tau^{-} \rightarrow \pi^{-} \pi^{-} \pi^{+} \nu_{\tau}\right)$ and in (32).

For the $\tau$ decay (23) to two pions, where both the charged and the neutral pion are measured, the respective decay density matrix is defined accordingly by integrating the corresponding squared matrix element over the four-momentum of the neutrino.

\footnotetext{
${ }^{2}$ Formula (4.3) of Ref. [9] contains a typo. These branching fraction factors are missing. However, they were taken into account in the numerical results given in that paper. Moreover, the variable $q_{0}^{*}$ on the l.h.s. of Eq. (4.4) of that reference should be replaced by $\left|\mathbf{q}^{*}\right| /\left\langle n_{A}\right\rangle$.
} 
In order to get the inclusive cross section for case (ii), considering, for instance, the decay of the $\tau^{-}$into three observed pions, we have to make in (30) the replacement

$$
\begin{aligned}
& \frac{d^{3} q_{-}}{(2 \pi)^{3} 2 q_{-}^{0}} \mathcal{D}_{\beta^{\prime} \beta}^{a}\left[\tau^{-} \rightarrow a+X\right] \\
& \rightarrow \prod_{i=1}^{3} \frac{d^{3} q_{i}}{(2 \pi)^{3} 2 q_{i}^{0}} \mathcal{D}_{\beta^{\prime} \beta}^{A}\left[\tau^{-} \rightarrow \pi^{-} \pi^{-} \pi^{+} \nu_{\tau}\right] .
\end{aligned}
$$

Analogous replacements apply if the decay of $\tau^{+}$to three observed pions or the decay of $\tau^{\mp}$ to two observed pions are analyzed.

The production density matrix $R$ in (30) is computed in the $e^{+} e^{-}$c.m. system; see below. Instead of calculating the decay density matrices also in this frame we can determine them in the $\tau^{-}$and $\tau^{+}$rest systems, respectively, if we use the following:

(i) We consider rotation-free Lorentz transformations (boosts) from the c.m. frame to the $\tau^{-}$and $\tau^{+}$rest systems, respectively.

(ii) We use standard spinors $u_{\beta}(k), v_{\alpha}(k)$ for the $\tau$ 's with $\beta, \alpha$ denoting the spin components in a given $z$ direction (see, e.g., [33]).

As is well known, these spin components are not changed by boost transformations. Let $\Lambda_{\mathbf{k}}$ be the boost transforming the $\tau^{-}$momentum $k_{-}$from the $e^{+} e^{-}$c.m. system to rest,
$\Lambda_{\mathbf{k}} k_{-}=k_{-}^{*}$, where $k_{-}^{*}=\left(m_{\tau}, \mathbf{0}\right)^{T}$. We have then with $\Lambda_{\mathbf{k}} q_{-}=q_{-}^{*}$; see (B2) and (B3),

$$
\left\langle a\left(q_{-}\right), X|\mathcal{T}| \tau^{-}\left(k_{-}, \beta\right)\right\rangle=\left\langle a\left(q_{-}^{*}\right), X|\mathcal{T}| \tau^{-}\left(k_{-}^{*}, \beta\right)\right\rangle .
$$

Insertion into the decay matrix (26) proves our statements above. The analogous argumentation applies to the $\tau^{+}$ decay density matrices.

In Appendix A we give the explicit forms of the $\tau^{\mp}$ decay density matrices in the respective rest frames for the decay modes listed in (22)-(24).

Finally, using the one-photon-exchange approximation and setting

$$
F_{1}\left(q^{2}\right)=1, \quad F_{2}\left(q^{2}\right)=0, \quad A\left(q^{2}\right)=0,
$$

the production density matrix $R$ is given in the $e^{+} e^{-}$c.m. frame by

$$
R=\frac{\chi}{\left|1+e^{2} \Pi_{c}(s)\right|^{2}},
$$

where [9]

$$
\chi=\chi_{\mathrm{SM}}+\operatorname{Re} \hat{d}_{\tau} \chi_{C P}^{R}+\operatorname{Im} \hat{d}_{\tau} \chi_{C P}^{I}+\chi_{\hat{d}^{2}},
$$

and

$$
\begin{aligned}
\chi_{\mathrm{SM}}= & \frac{e^{4}}{s}\left\{\left[k_{0}^{2}+m_{\tau}^{2}+|\mathbf{k}|^{2}(\hat{\mathbf{k}} \cdot \hat{\mathbf{p}})^{2}\right] \mathbb{1}-\left(\boldsymbol{\sigma}_{+} \cdot \boldsymbol{\sigma}_{-}\right)|\mathbf{k}|^{2}\left[1-(\hat{\mathbf{k}} \cdot \hat{\mathbf{p}})^{2}\right]\right. \\
& +2\left(\hat{\mathbf{k}} \cdot \boldsymbol{\sigma}_{+}\right)\left(\hat{\mathbf{k}} \cdot \boldsymbol{\sigma}_{-}\right)\left[|\mathbf{k}|^{2}+\left(k_{0}-m_{\tau}\right)^{2}(\hat{\mathbf{k}} \cdot \hat{\mathbf{p}})^{2}\right]+2 k_{0}^{2}\left(\hat{\mathbf{p}} \cdot \boldsymbol{\sigma}_{+}\right)\left(\hat{\mathbf{p}} \cdot \boldsymbol{\sigma}_{-}\right) \\
& \left.-2 k_{0}\left(k_{0}-m_{\tau}\right)(\hat{\mathbf{k}} \cdot \hat{\mathbf{p}})\left[\left(\hat{\mathbf{k}} \cdot \boldsymbol{\sigma}_{+}\right)\left(\hat{\mathbf{p}} \cdot \boldsymbol{\sigma}_{-}\right)+\left(\hat{\mathbf{k}} \cdot \boldsymbol{\sigma}_{-}\right)\left(\hat{\mathbf{p}} \cdot \boldsymbol{\sigma}_{+}\right)\right]\right\},
\end{aligned}
$$

$$
\begin{aligned}
\chi_{C P}^{R}= & -2 e^{4} \frac{|\mathbf{k}|}{s}\left\{-\left[m_{\tau}+\left(k_{0}-m_{\tau}\right)(\hat{\mathbf{k}} \cdot \hat{\mathbf{p}})^{2}\right]\left(\boldsymbol{\sigma}_{+} \times \boldsymbol{\sigma}_{-}\right) \cdot \hat{\mathbf{k}}\right. \\
& \left.+k_{0}(\hat{\mathbf{k}} \cdot \hat{\mathbf{p}})\left(\boldsymbol{\sigma}_{+} \times \boldsymbol{\sigma}_{-}\right) \cdot \hat{\mathbf{p}}\right\}, \\
\chi_{C P}^{I}= & 2 e^{4} \frac{|\mathbf{k}|}{s}\left\{-\left[m_{\tau}+\left(k_{0}-m_{\tau}\right)(\hat{\mathbf{k}} \cdot \hat{\mathbf{p}})^{2}\right]\left(\boldsymbol{\sigma}_{+}-\boldsymbol{\sigma}_{-}\right) \cdot \hat{\mathbf{k}}\right. \\
& \left.+k_{0}(\hat{\mathbf{k}} \cdot \hat{\mathbf{p}})\left(\boldsymbol{\sigma}_{+}-\boldsymbol{\sigma}_{-}\right) \cdot \hat{\mathbf{p}}\right\}, \\
\chi_{\hat{d}^{2}}= & e^{4}\left[\left(\operatorname{Re} \hat{d}_{\tau}\right)^{2}+\left(\operatorname{Im} \hat{d}_{\tau}\right)^{2}\right] \frac{|\mathbf{k}|^{2}}{s}\left[1-(\hat{\mathbf{k}} \cdot \hat{\mathbf{p}})^{2}\right]\left(\mathbb{1}-\boldsymbol{\sigma}_{+} \cdot \boldsymbol{\sigma}_{-}\right) .
\end{aligned}
$$

Compared to Eqs. (3.8)-(3.10) of [9] we neglect here the contributions from $Z$-boson exchange because we restrict ourselves to the kinematic range $s \ll m_{Z}^{2}$, but we have included the photon vacuum polarization effects. In (38)(41) we put $\mathbf{p}=\mathbf{p}_{+}, \mathbf{k}=\mathbf{k}_{+}$, and $\hat{\mathbf{p}}$ and $\hat{\mathbf{k}}$ denote the respective unit vectors; we have introduced in (37) and (41) dimensionless EDM form factors defined by

$\operatorname{Re} \hat{d}_{\tau}(s)=\frac{\sqrt{s}}{e} \operatorname{Re} d_{\tau}(s), \quad \operatorname{Im} \hat{d}_{\tau}(s)=\frac{\sqrt{s}}{e} \operatorname{Im} d_{\tau}(s)$.

Moreover, we use in the equations above the notation [9]

$$
\begin{aligned}
\mathbb{1} & \equiv(\mathbb{1} \otimes \mathbb{1})_{\alpha \alpha^{\prime} \beta \beta^{\prime}}=\delta_{\alpha \alpha^{\prime}} \delta_{\beta \beta^{\prime}}, \\
\boldsymbol{\sigma}_{+} & \equiv(\sigma \otimes \mathbb{1})_{\alpha \alpha^{\prime} \beta \beta^{\prime}}=\boldsymbol{\sigma}_{\alpha \alpha^{\prime}} \delta_{\beta \beta^{\prime}}, \\
\boldsymbol{\sigma}_{-} & \equiv(\mathbb{1} \otimes \boldsymbol{\sigma})_{\alpha \alpha^{\prime} \beta \beta^{\prime}}=\delta_{\alpha \alpha^{\prime}} \boldsymbol{\sigma}_{\beta \beta^{\prime}},
\end{aligned}
$$

where the first and second factors in these tensor products refer to the spin spaces of $\tau^{+}$and $\tau^{-}$, respectively. The density matrices $\chi_{\mathrm{SM}}$ and $\chi_{\hat{d}^{2}}$ are $C P$-even, whereas $\chi_{C P}^{R}$ is $C P$ - and $T_{N}$-odd while $\chi_{C P}^{I}$ is $C P$-odd and $T_{N}$-even. Here and below $T_{N}$-even/odd refers to the behavior with respect 
to the naive "time reversal" transformation, that is, reflections of three-momenta and spins.

Equation (39) shows that a nonzero $\operatorname{Re} d_{\tau}$ generates $C P$ odd $\tau^{+} \tau^{-}$spin correlations in the $\mathbf{p}, \mathbf{k}$ scattering plane while a nonzero imaginary part of $d_{\tau}$ leads to a $C P$-odd asymmetry of the $\tau^{+}$and $\tau^{-}$polarizations with projections along $\mathbf{p}$ and $\mathbf{k}$; cf. (40). The $\tau$ leptons autoanalyze their spin directions via their parity-violating weak decays. In this way these $\tau$ spin correlations and polarization asymmetries induce $C P$-odd angular correlations among the $\tau^{ \pm}$decay products, to which we now turn.

\section{SIMPLE AND OPTIMAL CP OBSERVABLES}

In this chapter we discuss simple and optimal observables for studying $C P$ violation in the reactions (5). Let us first consider the case (i) above where only one charged particle is measured from $\tau^{-}$and $\tau^{+}$decay, respectively, i.e., $\tau^{-} \rightarrow a\left(q_{-}\right)+X$ and $\tau^{+} \rightarrow \bar{b}\left(q_{+}\right)+X^{\prime}$. Simple $C P$ observables for this case were given in Ref. [9]. Observables sensitive to $\operatorname{Re} d_{\tau}(s)$ are, for instance, the tensors

$$
\begin{aligned}
& \hat{T}^{i j}=\left(\hat{\mathbf{q}}_{+}-\hat{\mathbf{q}}_{-}\right)^{i} \frac{\left(\hat{\mathbf{q}}_{+} \times \hat{\mathbf{q}}_{-}\right)^{j}}{\left|\hat{\mathbf{q}}_{+} \times \hat{\mathbf{q}}_{-}\right|}+(i \leftrightarrow j), \\
& T^{i j}=\left(\mathbf{q}_{+}-\mathbf{q}_{-}\right)^{i}\left(\mathbf{q}_{+} \times \mathbf{q}_{-}\right)^{j}+(i \leftrightarrow j) .
\end{aligned}
$$

Observables sensitive to $\operatorname{Im} d_{\tau}(s)$ are, for instance,

$$
\begin{gathered}
\hat{Q}^{i j}=\left(\hat{\mathbf{q}}_{+}+\hat{\mathbf{q}}_{-}\right)^{i}\left(\hat{\mathbf{q}}_{+}-\hat{\mathbf{q}}_{-}\right)^{j}+(i \leftrightarrow j), \\
Q^{i j}=\left(\mathbf{q}_{+}+\mathbf{q}_{-}\right)^{i}\left(\mathbf{q}_{+}-\mathbf{q}_{-}\right)^{j}-\frac{1}{3} \delta^{i j}\left(\mathbf{q}_{+}^{2}-\mathbf{q}_{-}^{2}\right)+(i \leftrightarrow j) .
\end{gathered}
$$

The momenta $\mathbf{q}_{\mp}$ in (44)-(47) are defined in the $e^{+} e^{-}$c.m. frame, and $\hat{\mathbf{q}}_{ \pm}=\mathbf{q}_{ \pm} /\left|\mathbf{q}_{ \pm}\right|$and $i, j \in\{1,2,3\}$ are the Cartesian vector indices. These observables, denoted generically by $\mathcal{O}\left(\mathbf{q}_{+}, \mathbf{q}_{-}\right)$, have the property to be odd under $C P$ :

$$
\mathcal{O}\left(\mathbf{q}_{+}, \mathbf{q}_{-}\right)=-\mathcal{O}\left(-\mathbf{q}_{-},-\mathbf{q}_{+}\right) .
$$

Moreover, Eqs. (44) and (45) are $T_{N}$-odd while (46) and (47) are $T_{N}$-even. A nonzero expectation value of any such observable of the form

$$
\begin{aligned}
\langle\mathcal{O}\rangle_{a b} & \equiv \frac{1}{2}\left\{\langle\mathcal{O}\rangle_{a \bar{b}}+\langle\mathcal{O}\rangle_{b \bar{a}}\right\} \\
& =\frac{1}{2}\left\{\frac{\int d \sigma_{a \bar{b}} \mathcal{O}}{\int d \sigma_{a \bar{b}}}+\frac{\int d \sigma_{b \bar{a}} \mathcal{O}}{\int d \sigma_{b \bar{a}}}\right\}
\end{aligned}
$$

is a genuine signature of $C P$ violation. Here $d \sigma_{a \bar{b}}$ is the cross section (30) of the reaction (28) and $d \sigma_{b \bar{a}}$ the corresponding one for the charge-conjugate channel. We assume that any phase-space cuts that may be applied are made in a $C P$-symmetric way.

Observables of the type (44)-(47) were studied extensively in [9]. In Chapter 5 we give an update of the sensitivities achievable with these observables at the KEKB accelerator with Belle II. A discussion of the sensitivities achievable with the BES III experiment at the Beijing Electron-Positron Collider II is deferred to a future publication.

We shall now turn to optimal observables [21-23], and we follow here Ref. [23]. We denote the measured phasespace variables generically by $\phi$ and the $C P$-transformed ones by $\bar{\phi}$ :

$$
C P: \phi \rightarrow \bar{\phi} .
$$

Phase-space cuts are assumed to be $C P$-symmetric. In the following we denote the dimensionless $C P$-violating EDM form factors [cf. Eq. (42)] that are to be measured by

$$
g_{1}=\operatorname{Re} \hat{d}_{\tau}, \quad g_{2}=\operatorname{Im} \hat{d}_{\tau} .
$$

From experiment we know that these couplings are small, $\left|g_{1,2}\right| \ll 1$. From (3) we get $\left|g_{1,2}\right| \leq 2.4 \times 10^{-2}$ for $\sqrt{s}=10.58 \mathrm{GeV}$. Therefore, we shall work to leading order in these couplings. The cross section (30) can be expanded in the $g_{i}$ as follows, neglecting terms of second order in these couplings:

$$
S^{a \bar{b}}(\phi)=\frac{d \sigma_{a \bar{b}}(\phi)}{d \phi}=S_{0}^{a \bar{b}}(\phi)+g_{i} S_{1, i}^{a \bar{b}}(\phi) .
$$

Here and in the following we use the summation convention. Moreover, in order not to overload the notation, the labels $a$ and $\bar{b}$ denote in (52) and in what follows decays of $\tau^{-}$and $\tau^{+}$to one, two, or three measured particles, respectively. The $C P$ properties of $S_{0}$ and $S_{1}$ in (52) are

$$
S_{0}^{a \bar{b}}(\phi)=S_{0}^{b \bar{a}}(\bar{\phi}), \quad S_{1, i}^{a \bar{b}}(\phi)=-S_{1, i}^{b \bar{a}}(\bar{\phi}) .
$$

We define now the observables

$$
\mathcal{O}_{i}^{a \bar{b}}(\phi)=S_{1, i}^{a \bar{b}}(\phi) / S_{0}^{a \bar{b}}(\phi) .
$$

Their expectation value $E_{0}$ for $g_{i}=0$ is

$$
E_{0}\left(\mathcal{O}_{i}^{a \bar{b}}\right)=\int d \phi S_{0}^{a \bar{b}}(\phi) \mathcal{O}_{i}^{a \bar{b}}(\phi) / \int d \phi^{\prime} S_{0}^{a \bar{b}}\left(\phi^{\prime}\right) .
$$

We set

$$
\mathcal{O}_{i}^{\prime a \bar{b}}(\phi)=\mathcal{O}_{i}^{a \bar{b}}(\phi)-E_{0}\left(\mathcal{O}_{i}^{a \bar{b}}\right)
$$

and get for the expectation value of $\mathcal{O}_{i}^{\prime a \bar{b}}$ : 


$$
\begin{aligned}
E\left(\mathcal{O}_{i}^{\prime a \bar{b}}\right) & =\int d \phi S^{a \bar{b}}(\phi) \mathcal{O}_{i}^{\prime a \bar{b}}(\phi) / \int d \phi^{\prime} S^{a \bar{b}}\left(\phi^{\prime}\right) \\
& =V_{i j}\left(\mathcal{O}^{\prime a \bar{b}}\right) g_{j} .
\end{aligned}
$$

The expression on the right-hand side is obtained by expanding the ratio to first order in the $g_{j}$. Here $V\left(\mathcal{O}^{\prime a \bar{b}}\right)=$ $\left(V_{i j}\left(\mathcal{O}^{\prime a \bar{b}}\right)\right)$ is the covariance matrix of the quantities $\mathcal{O}^{\prime}$ for $g_{j}=0$.

$$
\begin{aligned}
V_{i j}\left(\mathcal{O}^{\prime a \bar{b}}\right)= & E_{0}\left(\mathcal{O}_{i}^{\prime a \bar{b}} \mathcal{O}_{j}^{\prime a \bar{b}}\right)=E_{0}\left(\frac{S_{1, i}^{a \bar{b}}}{S_{0}^{a \bar{b}}} \frac{S_{1, j}^{a \bar{b}}}{S_{0}^{a \bar{b}}}\right) \\
& -E_{0}\left(\frac{S_{1, i}^{a \bar{b}}}{S_{0}^{a \bar{b}}}\right) E_{0}\left(\frac{S_{1, j}^{a \bar{b}}}{S_{0}^{a \bar{b}}}\right) .
\end{aligned}
$$

The covariance matrix $V\left(\mathcal{O}^{\prime a \bar{b}}\right)$ is positive definite. From (57) we obtain

$$
g_{i}=V_{i j}^{-1}\left(\mathcal{O}^{\prime a \bar{b}}\right) E\left(\mathcal{O}_{j}^{\prime a \bar{b}}\right) .
$$

In the remainder of this section we recall from [23] some general relations for optimal observables in order to make our article self-contained. Also, we shall discuss that in the nondiagonal case $a \neq b$ the theoretically optimal estimators may not always be "optimal" from a practical point of view [see the discussion after Eq. (78) below].

We consider first the diagonal case, $a=b$, and assume that $n$ events of this type are analyzed. The density function is then

$$
\begin{aligned}
F\left(\phi_{1}, \ldots, \phi_{n}\right) & =\prod_{k=1}^{n} f\left(\phi_{k}\right), \\
f(\phi) & =S^{a \bar{a}}(\phi) / \int d \phi^{\prime} S^{a \bar{a}}\left(\phi^{\prime}\right) .
\end{aligned}
$$

The information matrix $I=\left(I_{i j}\right)$ is defined by

$$
I_{i j}=E\left[\left(\frac{\partial}{\partial g_{i}} \ln F\right)\left(\frac{\partial}{\partial g_{j}} \ln F\right)\right] .
$$

The optimal estimators for the couplings $g_{i}$ are in this case

$$
\gamma_{i}(\phi)=V_{i j}^{-1}\left(\mathcal{O}^{\prime a \bar{a}}\right) \overline{\mathcal{O}}_{j}^{a \bar{a}}(\phi),
$$

where $\overline{\mathcal{O}_{j}^{\prime}}$ denotes the mean value of $\mathcal{O}_{j}^{\prime}$. From Eqs. (59) and (60) we obtain the expectation values

$$
E\left(\gamma_{i}\right)=g_{i},
$$

and the covariance matrix of the $\gamma_{i}$, evaluated for $g_{i}=0$, is

$$
V_{i j}(\gamma)=E_{0}\left(\gamma_{i} \gamma_{j}\right)=\frac{1}{n} V_{i j}^{-1}\left(\mathcal{O}^{\prime a \bar{a}}\right)
$$

We get for the information matrix (61)

$$
\left.I\right|_{g=0}=n V\left(\mathcal{O}^{\prime a \bar{a}}\right) .
$$

Therefore, we have here

$$
V^{-1}(\gamma)=\left.I\right|_{g=0},
$$

and the estimators (62) are optimal for small $g_{j}$. That is, the error ellipse obtained with the estimators $\gamma_{i}$ in (62) is given by the one obtained from $I$ which is the smallest one possible. We note that due to the $C P$ properties (53) of $S_{0}$ and $S_{1, i}$ we have in the diagonal case $a=b$, assuming possible cuts in phase space to be $C P$-symmetric:

$E_{0}\left(\mathcal{O}_{i}^{a \bar{a}}\right)=0, \quad \mathcal{O}_{i}^{\prime a \bar{a}}(\phi)=\mathcal{O}_{i}^{a \bar{a}}(\phi), \quad V\left(\mathcal{O}^{\prime a \bar{a}}\right)=V\left(\mathcal{O}^{a \bar{a}}\right)$,

and the optimal estimators are

$$
\gamma_{i}(\phi)=V_{i j}^{-1}\left(\mathcal{O}^{a \bar{a}}\right) \overline{\mathcal{O}}_{j}^{a \bar{a}}(\phi) ;
$$

see (55), (56), and (62).

Finally, we treat the nondiagonal case, $a \neq b$. We assume that any phase-space cuts made for the channel $a \bar{b}$ are applied to $b \bar{a}$ in a $C P$-conjugate way. We get then from the $C P$ relations (53)

$$
\begin{aligned}
\int d \phi S_{0}^{a \bar{b}}(\phi) & =\int d \bar{\phi} S_{0}^{b \bar{a}}(\bar{\phi}), \\
E_{0}\left(\frac{S_{1, i}^{a \bar{b}}}{S_{0}^{a \bar{b}}}\right) & =-E_{0}\left(\frac{S_{1, i}^{b \bar{a}}}{S_{0}^{b \bar{a}}}\right), \\
V\left(\mathcal{O}^{\prime a \bar{b}}\right) & =V\left(\mathcal{O}^{\prime b \bar{a}}\right) .
\end{aligned}
$$

We assume that $n_{1}$ events of the type $a \bar{b}$ and $n_{2}$ events $b \bar{a}$ are analyzed. The density function is then

$F\left(\phi_{1}, \ldots, \phi_{n_{1}}, \bar{\phi}_{1}, \ldots, \bar{\phi}_{n_{2}}\right)=\prod_{k=1}^{n_{1}} f_{a \bar{b}}\left(\phi_{k}\right) \prod_{l=1}^{n_{2}} f_{b \bar{a}}\left(\bar{\phi}_{l}\right)$

with

$$
\begin{aligned}
& f_{a \bar{b}}(\phi)=S^{a \bar{b}}(\phi) / \int d \phi^{\prime} S^{a \bar{b}}\left(\phi^{\prime}\right), \\
& f_{b \bar{a}}(\bar{\phi})=S^{b \bar{a}}(\bar{\phi}) / \int d \bar{\phi}^{\prime} S^{b \bar{a}}\left(\bar{\phi}^{\prime}\right) .
\end{aligned}
$$

Here the information matrix $I=\left(I_{i j}\right)$ is given for $g_{i}=0$ by 
$\left.I_{i j}\right|_{g=0}=\left.E\left[\left(\frac{\partial}{\partial g_{i}} \ln F\right)\left(\frac{\partial}{\partial g_{j}} \ln F\right)\right]\right|_{g=0}=n V_{i j}\left(\mathcal{O}^{\prime a \bar{b}}\right)$,

where $n=n_{1}+n_{2}$. Here it is convenient to use as estimators for the couplings $g_{i}$, with $\mathcal{O}_{j}$ from (54):

$\gamma_{i}(\phi, \bar{\phi})=\frac{1}{4}\left[V_{i j}^{-1}\left(\mathcal{O}^{\prime a \bar{b}}\right)+V_{i j}^{-1}\left(\mathcal{O}^{\prime b \bar{a}}\right)\right]\left[\overline{\mathcal{O}}_{j}^{a \bar{b}}(\phi)+\overline{\mathcal{O}}_{j}^{b \bar{a}}(\bar{\phi})\right]$.

We have

$$
\begin{aligned}
E\left(\frac{1}{2} \overline{\mathcal{O}}_{i}^{a \bar{b}}+\frac{1}{2} \overline{\mathcal{O}}_{i}^{b \bar{a}}\right) & =E\left(\frac{1}{2} \mathcal{O}_{i}^{a \bar{b}}+\frac{1}{2} \mathcal{O}_{i}^{b \bar{a}}\right)=V_{i j}\left(\mathcal{O}^{\prime a \bar{b}}\right) g_{j} \\
E\left(\gamma_{i}\right) & =V_{i k}^{-1}\left(\mathcal{O}^{\prime a \bar{b}}\right) V_{k j}\left(\mathcal{O}^{\prime a \bar{b}}\right) g_{j}=g_{i}
\end{aligned}
$$

The covariance matrix of these estimators is obtained as

$$
V(\gamma)=\frac{n_{1}+n_{2}}{4 n_{1} n_{2}} V^{-1}\left(\mathcal{O}^{\prime a \bar{b}}\right),
$$

which implies

$$
\begin{aligned}
V^{-1}(\gamma) & =n\left(1-\frac{\left(n_{1}-n_{2}\right)^{2}}{n^{2}}\right) V\left(\mathcal{O}^{\prime a \bar{b}}\right) \\
& =\left.\left(1-\frac{\left(n_{1}-n_{2}\right)^{2}}{n^{2}}\right) I\right|_{g=0} .
\end{aligned}
$$

The $\gamma_{i}$ in Eq. (75) are the optimal estimators for $n_{1}=n_{2}=n / 2$. For $n_{1} \neq n_{2}$ they are not quite optimal, but for the theoretically optimal estimators one would need in this case the precise knowledge of $E_{0}\left(S_{1, i}^{a \bar{b}} / S_{0}^{a \bar{b}}\right)$. This would introduce an unnecessary source of uncertainty in the measurements.

To conclude this section we remark on the following. A more elaborate description of $\tau$-pair production and decay would take higher-order radiative corrections into account. Let us denote the resulting differential cross section by $\tilde{S}^{a \bar{b}}$,

$$
\frac{d \sigma_{a \bar{b}}}{d \phi}(\phi)=\tilde{S}^{a \bar{b}}(\phi)
$$

If it is $C P$-invariant, we have

$$
\tilde{S}^{a \bar{b}}(\phi)=\tilde{S}^{b \bar{a}}(\bar{\phi}) .
$$

Then the corresponding expectation values $\tilde{E}$ of the estimators $\gamma_{i}$ defined in (68) and (75) and constructed with the expressions $S_{0}, S_{1, i}$ from (52) will, of course, be zero due to $(53)$ :

$$
\tilde{E}\left(\gamma_{i}\right)=0
$$

That is, the observables $\gamma_{i}$ given in (68) and (75) are in all cases genuine $C P$ observables. They cannot get nonzero expectation values, neither from $C P$-conserving radiative $\mathrm{SM}$ corrections nor from $C P$-conserving interactions beyond the SM.

\section{NUMERICAL RESULTS AT $\sqrt{s}=10.58 \mathrm{GeV}$}

We consider now $\tau$-pair production and decay at the $\Upsilon(4 \mathrm{~S})$ resonance at $\sqrt{s}=10.58 \mathrm{GeV}$ and compute the expectation values of the simple and optimal $C P$ observables discussed in the previous section and estimate the resulting 1 s.d. (standard deviation) statistical sensitivities to the EDM form factors $\operatorname{Re} d_{\tau}$ and $\operatorname{Im} d_{\tau}$ at this c.m. energy. The expectation values of the $C P$ observables are computed to leading order in the real and imaginary parts of the $\tau$ EDM form factor using the expression (30) for the differential cross section with (35)-(40) and several of the decay density matrices given in Appendix A. First, no phase-space cuts are applied. At the end of this section we analyze also the effects of cuts.

The expectation values of the observables (44)-(47) at the $\Upsilon(4 \mathrm{~S})$ resonance in the decay channels where only one charged particle from $\tau^{-}$and one from $\tau^{+}$decay is measured [case (i) above] are of the form

$$
\begin{gathered}
\left\langle T^{i j}\right\rangle_{a b}=c_{a b}(s) \operatorname{Re} \hat{d}_{\tau}(s) s^{i j}, \quad\left\langle\hat{T}^{i j}\right\rangle_{a b}=\tilde{c}_{a b}(s) \operatorname{Re} \hat{d}_{\tau}(s) s^{i j}, \\
\left\langle Q^{i j}\right\rangle_{a b}=\kappa_{a b}(s) \operatorname{Im} \hat{d}_{\tau}(s) s^{i j}, \\
\left\langle\hat{Q}^{i j}\right\rangle_{a b}=\tilde{\kappa}_{a b}(s) \operatorname{Im} \hat{d}_{\tau}(s) s^{i j} .
\end{gathered}
$$

In the case of nondiagonal decay channels $a \neq b$ the expectation values are calculated as averages defined in (49). The expectation values of the symmetric traceless tensors (44)-(47) must be proportional to a tensor $s^{i j}$ with the same property. Using the $e^{+}$beam direction $\hat{\mathbf{p}}$ in the $e^{+} e^{-}$c.m. frame we have

$$
\left(s^{i j}\right)=\frac{1}{2}\left(\hat{p}^{i} \hat{p}^{j}-\frac{1}{3} \delta^{i j}\right)=\operatorname{diag}\left(-\frac{1}{6},-\frac{1}{6}, \frac{1}{3}\right) .
$$

The right-hand side of (84) follows from identifying $\mathbf{p}$ with the $z$ axis which we do in the following. Equation (84) is identical to the tensor polarization of the intermediate photon state. Because the diagonal elements of the above tensor observables are not independent, we consider only their 3,3 components that have the largest expectation values. Naive "time reversal" invariance $T_{N}$ implies that the expectation values (82) and (83) do not depend on $\operatorname{Im} \hat{d}_{\tau}$ and $\operatorname{Re} \hat{d}_{\tau}$, respectively. That is, the covariance matrix of the $T$ and $Q$ tensors is diagonal; see Appendix B.

In order to estimate the statistical error in the measurement of the expectation values of the observables $\mathcal{O}$ we compute also the respective standard deviation $\Delta \mathcal{O}=$ $\sqrt{\left\langle\mathcal{O}^{2}\right\rangle-\langle\mathcal{O}\rangle^{2}}$ of the distribution of $\mathcal{O}$ in the SM for 
TABLE II. Observables $T^{i j}$ and $\hat{T}^{i j}$ at $\sqrt{s}=10.58 \mathrm{GeV}\left(N_{\tau \tau}=4.5 \times 10^{10}\right)$.

\begin{tabular}{lccccccc}
\hline \hline$\tau^{-} \rightarrow$ & $\tau^{+} \rightarrow$ & $c_{a b}\left[\mathrm{GeV}^{3}\right]$ & $\sqrt{\left\langle T_{33}^{2}\right\rangle_{a b}}\left[\mathrm{GeV}^{3}\right]$ & $\delta \operatorname{Re} d_{\tau}\left(\times 10^{-19} e \mathrm{~cm}\right)$ & $\tilde{c}_{a b}$ & $\sqrt{\left\langle\hat{T}_{33}^{2}\right\rangle_{a b}}$ & $\delta \operatorname{Re} d_{\tau}\left(\times 10^{-19} e \mathrm{~cm}\right)$ \\
\hline$\pi^{-} \nu$ & $\pi^{+} \bar{\nu}$ & 4.46 & 11.34 & 6.21 & 0.332 & 1.02 & 7.50 \\
$\rho^{-} \nu$ & $\rho^{+} \bar{\nu}$ & 0.71 & 10.07 & 14.7 & 0.043 & 1.06 & 25.5 \\
$\pi^{-} \nu$ & $\rho^{+} \bar{\nu}$ & 1.79 & 10.71 & 6.74 & 0.110 & 1.03 & 10.5 \\
$\ell^{-} \nu \bar{\nu}$ & $\ell^{+1} \bar{\nu} \nu$ & 0.36 & 4.68 & 9.86 & 0.037 & 0.98 & 19.9 \\
$\ell^{-} \nu \bar{\nu}$ & $\pi^{+} \bar{\nu}$ & -1.27 & 6.66 & 5.05 & -0.111 & 0.96 & 8.3 \\
$\ell^{-} \nu \bar{\nu}$ & $\rho^{+} \bar{\nu}$ & -0.51 & 6.78 & 8.32 & -0.037 & 1.00 & 16.9 \\
\hline \hline
\end{tabular}

TABLE III. Observables $Q^{i j}$ and $\hat{Q}^{i j}$ at $\sqrt{s}=10.58 \mathrm{GeV}\left(N_{\tau \tau}=4.5 \times 10^{10}\right)$.

\begin{tabular}{lccccccc}
\hline \hline$\tau^{-} \rightarrow$ & $\tau^{+} \rightarrow$ & $\kappa_{a b}\left[\mathrm{GeV}^{3}\right]$ & $\sqrt{\left\langle Q_{33}^{2}\right\rangle_{a b}}\left[\mathrm{GeV}^{3}\right]$ & $\delta \operatorname{Im} d_{\tau}\left(\times 10^{-19} e \mathrm{~cm}\right)$ & $\tilde{\kappa}_{a b}$ & $\sqrt{\left\langle\hat{Q}_{33}^{2}\right\rangle_{a b}}$ & $\delta \operatorname{Im} d_{\tau}\left(\times 10^{-19} e \mathrm{~cm}\right)$ \\
\hline$\pi^{-} \nu$ & $\pi^{+} \bar{\nu}$ & -5.26 & 6.56 & 3.04 & -0.601 & 0.59 & 2.38 \\
$\rho^{-} \nu$ & $\rho^{+} \bar{\nu}$ & -2.28 & 7.01 & 3.18 & -0.171 & 0.34 & 2.05 \\
$\pi^{-} \nu$ & $\rho^{+} \bar{\nu}$ & -3.77 & 7.07 & 2.11 & -0.386 & 0.52 & 1.52 \\
$\ell^{-} \nu \bar{\nu}$ & $\ell^{\prime+} \bar{\nu} \nu$ & 1.40 & 4.90 & 2.64 & 0.201 & 0.64 & 2.40 \\
$\ell^{-} \nu \bar{\nu}$ & $\pi^{+} \bar{\nu}$ & -1.93 & 7.24 & 3.61 & -0.200 & 0.65 & 3.14 \\
$\ell^{-} \nu \bar{\nu}$ & $\rho^{+} \bar{\nu}$ & -0.44 & 7.32 & 10.4 & 0.015 & 0.54 & 22.5 \\
\hline \hline
\end{tabular}

the various decay channels. As discussed in Appendix B the SM expectation values of the tensors $T^{i j}, \hat{T}^{i j}$ vanish for the differential cross section as used by us. (Cf. Sec. III.) For the tensors $Q^{i j}, \hat{Q}^{i j}$ this is also true in the diagonal case $a=b$. In the nondiagonal case, $a \neq b$, their SM expectation values need not be zero, but are found numerically to be negligibly small. In Tables II and III we assume that the momenta of $\rho^{\mp}$ mesons can be experimentally determined, and we treat them as on-shell particles with the $\tau$-spin analyzing power given in (A10). The symbols $\ell$ and $\ell^{\prime}$ denote either the electron or muon, both are taken to be massless. We sum over the diagonal and nondiagonal $\ell \ell^{\prime}$ channels for estimating the respective sensitivity to the real and imaginary parts of the $\tau$ EDM. In a diagonal decay channel the number of events is $N_{a a}=N_{\tau \tau}(\operatorname{Br}(\tau \rightarrow a))^{2}$, while for a nondiagonal channel including its charge-conjugate mode we have $N_{a b}=2 N_{\tau \tau} \operatorname{Br}(\tau \rightarrow a) \operatorname{Br}(\tau \rightarrow b)$. The $\tau$ branching ratios are taken from [1]. We assume that the Belle II experiment will eventually record $N_{\tau \tau}=4.5 \times$ $10^{10} \tau$ pairs [19]. Considering as an example the measurements of $T_{33}$ and $Q_{33}$ in the decay channels $a \bar{b}$ and $b \bar{a}$ the resulting ideal 1 s.d. statistical errors of the dimensionful EDM couplings $\operatorname{Re} d_{\tau}$ and $\operatorname{Im} d_{\tau}$ are given by

$$
\begin{aligned}
\delta \operatorname{Re} d_{\tau}(s) & =\frac{e}{\sqrt{s}} \frac{1}{\sqrt{N_{a b}}} \frac{3\left[\left\langle T_{33}^{2}\right\rangle_{a b}\right]^{1 / 2}}{\left|c_{a b}\right|}, \\
\delta \operatorname{Im} d_{\tau}(s) & =\frac{e}{\sqrt{s}} \frac{1}{\sqrt{N_{a b}}} \frac{3\left[\left\langle Q_{33}^{2}\right\rangle_{a b}\right]^{1 / 2}}{\left|\kappa_{a b}\right|} .
\end{aligned}
$$

Equation (85) yields the absolute value that $\operatorname{Re} d_{\tau}\left(\operatorname{Im} d_{\tau}\right)$ must have in order that $\left\langle T_{33}\right\rangle_{a b}\left(\left\langle Q_{33}\right\rangle_{a b}\right)$ deviates from its SM prediction, namely zero, by 1 s.d. obtained from the square root of its SM variance. Formulas analogous to (85) hold for the dimensionless observables $\hat{T}_{33}$ and $\hat{Q}_{33}$.

Tables II and III contain our results for the expectation values [as defined in Eqs. (82) and (83)] and square roots of the variances of the observables (44)-(47) for several oneprong decays of $\tau^{\mp}$ where the charged particle has a sizable $\tau$-spin analyzing power. Moreover, the resulting 1 s.d. sensitivities to the real and imaginary parts of the $\tau$ EDM form factor are listed. ${ }^{3}$ Results for $T^{i j}$ and $\hat{Q}^{i j}$ were previously given in [9] and agree with those in Tables II and III. The accuracies $\delta \operatorname{Re} d_{\tau}$ and $\delta \operatorname{Im} d_{\tau}$ attainable in the various $\tau^{\mp}$ decay channels listed in Tables II and III show that the dimensionful observable $T_{33}$ is more sensitive than $\hat{T}_{33}$, while in the case of $Q_{33}$ and $\hat{Q}_{33}$ it is the other way around - except for the $\ell \rho$ decay channel which has, in any case, a rather poor sensitivity compared to the other decay modes.

Next we apply the optimal observables (54) for measuring $\operatorname{Re} \hat{d}_{\tau}$ and $\operatorname{Im} \hat{d}_{\tau}$ to the reactions of Sec. III. As in Eq. (52) and in the following equations, the labels $a, b$ refer here to the decays of $\tau^{-}$and/or $\tau^{+}$to one, two, or three measured particles. In particular, we take now the

\footnotetext{
${ }^{3}$ The last digit of the expectation values and variances listed in Tables II, III, and IV is rounded. The sensitivities $\delta \operatorname{Re} d_{\tau}$ and $\delta \operatorname{Im} d_{\tau}$ listed in these tables are computed with these rounded numbers.
} 
differential decay density matrices for $\tau \rightarrow 2 \pi \nu_{\tau}$ and $\tau \rightarrow$ $3 \pi \nu_{\tau}$ given in Appendix A into account. Using (38), (39), and (40) and the respective decay matrices $\mathcal{D}^{a}$ and $\mathcal{D}^{\bar{b}}$ we define

$\mathcal{O}_{R}^{a \bar{b}}=\frac{\operatorname{Tr}\left[\chi_{C P}^{R} \mathcal{D}^{a} \mathcal{D}^{\bar{b}}\right]}{\operatorname{Tr}\left[\chi_{\mathrm{SM}} \mathcal{D}^{a} \mathcal{D}^{\bar{b}}\right]}, \quad \mathcal{O}_{I}^{a \bar{b}}=\frac{\operatorname{Tr}\left[\chi_{C P}^{I} \mathcal{D}^{a} \mathcal{D}^{\bar{b}}\right]}{\operatorname{Tr}\left[\chi_{\mathrm{SM}} \mathcal{D}^{a} \mathcal{D}^{\bar{b}}\right]}$,

where the trace is taken with respect to the spin indices of $\tau^{-}$and $\tau^{+}$. Both observables are $C P$-odd and $\mathcal{O}_{R}^{a \bar{b}}$ is also $T_{N}$-odd while $\mathcal{O}_{I}^{a \bar{b}}$ is $T_{N}$-even. As already emphasized we compute the expectation values by integrating over the whole phase space. According to the general theory discussed in Sec. IV and Appendix B the covariance matrix for a decay channel $a \bar{b}$ is given, for zero $\tau$ EDM, by (58), (71):

$V\left(\mathcal{O}^{\prime a \bar{b}}\right)=V\left(\mathcal{O}^{\prime b \bar{a}}\right)=\left(\begin{array}{ll}E_{0}\left(\mathcal{O}_{R}^{\prime a \bar{b}} \mathcal{O}_{R}^{\prime a \bar{b}}\right) & E_{0}\left(\mathcal{O}_{R}^{\prime a \bar{b}} \mathcal{O}_{I}^{\prime a \bar{b}}\right) \\ E_{0}\left(\mathcal{O}_{I}^{\prime a \bar{b}} \mathcal{O}_{R}^{\prime a \bar{b}}\right) & E_{0}\left(\mathcal{O}_{I}^{\prime a \bar{b}} \mathcal{O}_{I}^{\prime a \bar{b}}\right)\end{array}\right)$,

where

$E_{0}\left(\mathcal{O}_{R}^{\prime a \bar{b}} \mathcal{O}_{R}^{\prime a \bar{b}}\right) \equiv\left\langle\left(\mathcal{O}_{R}^{\prime a \bar{b}}\right)^{2}\right\rangle_{0}, \quad E_{0}\left(\mathcal{O}_{I}^{\prime a \bar{b}} \mathcal{O}_{I}^{\prime a \bar{b}}\right) \equiv\left\langle\left(\mathcal{O}_{I}^{\prime a \bar{b}}\right)^{2}\right\rangle_{0}$,

etc., denote the expectation values for $d_{\tau}=0$. The expectation values for nonzero $\tau$ EDM are given by (76):

$$
\left(\begin{array}{c}
E\left(\frac{1}{2} \mathcal{O}_{R}^{a \bar{b}}+\frac{1}{2} \mathcal{O}_{R}^{b \bar{a}}\right) \\
E\left(\frac{1}{2} \mathcal{O}_{I}^{a \bar{b}}+\frac{1}{2} \mathcal{O}_{I}^{b \bar{a}}\right)
\end{array}\right) \equiv\left(\begin{array}{c}
\left\langle\mathcal{O}_{R}^{a b}\right\rangle \\
\left\langle\mathcal{O}_{I}^{a b}\right\rangle
\end{array}\right)=V\left(\mathcal{O}^{\prime a \bar{b}}\right)\left(\begin{array}{c}
\operatorname{Re} \hat{d}_{\tau}(s) \\
\operatorname{Im} \hat{d}_{\tau}(s)
\end{array}\right)
$$

We get for the covariance matrix of the optimal estimators of $\operatorname{Re} \hat{d}_{\tau}(s)$ and $\operatorname{Im} \hat{d}_{\tau}(s)$ [see (62), (64) and (75), (77)]

$$
V(\gamma)=\frac{1}{N_{a b}} V^{-1}\left(\mathcal{O}^{\prime a \bar{b}}\right) .
$$

Here $N_{a b}$ is the number of events in the diagonal channels $a=b$, whereas for $a \neq b$ it is the sum of the events $a \bar{b}$ and $\bar{b} a$, assuming that their numbers are equal.

However, with the form of the differential cross section used in this paper considerable simplifications occur. In the case where the $\tau$ leptons decay to one measured particle and/or to $2 \pi \nu_{\tau}$ where both pions are measured we have, as shown in Appendix B,

$\left\langle\mathcal{O}_{i}^{a \bar{b}}\right\rangle_{0}=0, \quad \mathcal{O}_{i}^{\prime a \bar{b}}=\mathcal{O}_{i}^{a \bar{b}} \quad(i=R, I), \quad\left\langle\mathcal{O}_{R}^{a \bar{b}} \mathcal{O}_{I}^{a \bar{b}}\right\rangle_{0}=0$.
That is, for these channels the respective covariance matrix (87) is diagonal.

When $\tau^{-}, \tau^{+}$, or both $\tau$ leptons decay to three measured pions, $\left\langle\mathcal{O}_{i}^{a \bar{b}}\right\rangle_{0}=0(i=R, I)$ still holds in the one-photon approximation (see Appendix B), but the covariance matrix is no longer diagonal. Yet we find for these decay modes that $\left\langle\mathcal{O}_{R}^{a \bar{b}} \mathcal{O}_{I}^{a \bar{b}}\right\rangle_{0}<$ a few $\times 10^{-4}$ with numerical uncertainties below $10^{-3}$. Therefore, within the precision of our numerical analysis the relations (91) hold also for these decay channels, and (89) simplifies to ${ }^{4}$

$$
\left\langle\mathcal{O}_{R}^{a b}\right\rangle=w_{a \bar{b}}(s) \operatorname{Re} \hat{d}_{\tau}(s), \quad\left\langle\mathcal{O}_{I}^{a b}\right\rangle=\omega_{a \bar{b}}(s) \operatorname{Im} \hat{d}_{\tau}(s),
$$

where we used the abbreviations

$$
w_{a \bar{b}} \equiv\left\langle\left(\mathcal{O}_{R}^{a \bar{b}}\right)^{2}\right\rangle_{0}, \quad \omega_{a \bar{b}} \equiv\left\langle\left(\mathcal{O}_{I}^{a \bar{b}}\right)^{2}\right\rangle_{0} .
$$

The resulting 1 s.d. errors of the dimensionful EDM couplings $\operatorname{Re} d_{\tau}$ and $\operatorname{Im} d_{\tau}$ are given by

$$
\begin{aligned}
\delta \operatorname{Re} d_{\tau}(s) & =\frac{e}{\sqrt{s}} \frac{1}{\sqrt{N_{a b}}} \frac{1}{\sqrt{\left\langle\left(\mathcal{O}_{R}^{a \bar{b}}\right)^{2}\right\rangle_{0}}}, \\
\delta \operatorname{Im} d_{\tau}(s) & =\frac{e}{\sqrt{s}} \frac{1}{\sqrt{N_{a b}}} \frac{1}{\sqrt{\left\langle\left(\mathcal{O}_{I}^{a \bar{b}}\right)^{2}\right\rangle_{0}}} .
\end{aligned}
$$

Table IV contains our results for the expectation values defined in Eq. (92) and for the square roots of the variances of the observables (86) for several $\tau^{\mp}$ decays to one, two, and/or three measured particles. The numbers in this table show that taking into account the full kinematic information on the hadronic system in the $\tau \rightarrow 2 \pi \nu_{\tau}$ and $\tau \rightarrow 3 \pi \nu_{\tau}$ decays results in maximal $\tau$-spin analyzing power [34,35], as is the case in the decay $\tau \rightarrow \pi \nu_{\tau}$. In addition, the resulting 1 s.d. sensitivities to the real and imaginary parts of the $\tau$ EDM form factor are given in Table IV, assuming again $4.5 \times 10^{10} \tau$-pair events. The 1 s.d. statistical errors $\delta \operatorname{Re} d_{\tau}$ and $\delta \operatorname{Im} d_{\tau}$ exhibited in Table IV signify that taking into account the channels where one or both $\tau$ leptons decay to two and/or three measured pions yields a significant improvement in the sensitivity to the $\tau$ EDM form factor. Comparing for each channel the accuracies $\delta \operatorname{Re} d_{\tau}$ and $\delta \operatorname{Im} d_{\tau}$ exhibited in Table IV with those in Tables II and III shows that, as expected, the optimal observables (86) are significantly more sensitive to the $\tau$ EDM than the observables $T_{33}$ and $\hat{Q}_{33}$.

If the measurement errors of the various exclusive $\tau^{+} \tau^{-}$ decay modes are uncorrelated, we may add in quadrature the statistical errors of $\operatorname{Re} d_{\tau}$ and $\operatorname{Im} d_{\tau}$ attainable for each channel:

\footnotetext{
${ }^{4}$ The left-hand sides of (92) denote averages according to (49).
} 
TABLE IV. Optimal observables $\mathcal{O}_{R}^{a \bar{b}}$ and $\mathcal{O}_{I}^{a \bar{b}}$ at $\sqrt{s}=10.58 \mathrm{GeV}\left(N_{\tau \tau}=4.5 \times 10^{10}\right)$.

\begin{tabular}{lccccccc}
\hline \hline$\tau^{-} \rightarrow$ & $\tau^{+} \rightarrow$ & $w_{a \bar{b}}$ & $\sqrt{\left\langle\left(\mathcal{O}_{R}^{a \bar{b}}\right)^{2}\right\rangle_{0}}$ & $\delta \operatorname{Re} d_{\tau}\left(\times 10^{-19} e \mathrm{~cm}\right)$ & $\omega_{a \bar{b}}$ & $\sqrt{\left\langle\left(\mathcal{O}_{I}^{a \bar{b}}\right)^{2}\right\rangle_{0}}$ & $\delta \operatorname{Im} d_{\tau}\left(\times 10^{-19} e \mathrm{~cm}\right)$ \\
\hline$\pi^{-} \nu$ & $\pi^{+} \bar{\nu}$ & 0.111 & 0.333 & 2.45 & 0.352 & 0.593 & 1.37 \\
$\pi^{-} \pi^{0} \nu$ & $\pi^{+} \pi^{0} \bar{\nu}$ & 0.111 & 0.333 & 1.04 & 0.352 & 0.593 & 0.58 \\
$\pi^{-} \pi^{-} \pi^{+} \nu$ & $\pi^{+} \pi^{+} \pi^{-} \bar{\nu}$ & 0.111 & 0.333 & 2.84 & 0.352 & 0.593 & 1.59 \\
$\pi^{-} \nu$ & $\pi^{+} \pi^{0} \bar{\nu}$ & 0.111 & 0.333 & 1.13 & 0.352 & 0.593 & 0.63 \\
$\pi^{-} \nu$ & $\pi^{+} \pi^{+} \pi^{-} \bar{\nu}$ & 0.111 & 0.333 & 1.86 & 0.352 & 0.593 & 1.05 \\
$\pi^{-} \pi^{0} \nu$ & $\pi^{+} \pi^{+} \pi^{-} \bar{\nu}$ & 0.111 & 0.333 & 1.21 & 0.352 & 0.593 & 0.68 \\
$\ell^{-} \nu \bar{\nu}$ & $\ell^{\prime+} \bar{\nu} \nu$ & 0.004 & 0.064 & 4.04 & 0.055 & 0.235 & 1.08 \\
$\ell^{-} \nu \bar{\nu}$ & $\pi^{+} \bar{\nu}$ & 0.020 & 0.142 & 2.26 & 0.162 & 0.402 & 0.80 \\
$\ell^{-} \nu \bar{\nu}$ & $\pi^{+} \pi^{0} \bar{\nu}$ & 0.020 & 0.142 & 1.47 & 0.162 & 0.402 & 0.52 \\
$\ell^{-} \nu \bar{\nu}$ & $\pi^{+} \pi^{+} \pi^{-} \bar{\nu}$ & 0.020 & 0.142 & 2.43 & 0.162 & 0.402 & 0.86 \\
\hline \hline
\end{tabular}

TABLE V. Ideal 1 s.d. statistical errors on $\operatorname{Re} d_{\tau}$ and $\operatorname{Im} d_{\tau}$ that result from adding the respective uncertainties attainable in the various decay channels in quadrature.

\begin{tabular}{|c|c|c|c|c|c|}
\hline \multirow{2}{*}{$\frac{\delta \operatorname{Re} d_{\tau}[e \mathrm{~cm}]}{\left\langle T_{33}\right\rangle_{a b}}$} & \multicolumn{5}{|c|}{$\delta \operatorname{Im} d_{\tau}[e \mathrm{~cm}]$} \\
\hline & $\begin{array}{c}\left\langle\hat{T}_{33}\right\rangle_{a b} \\
4.53 \times 10^{-19}\end{array}$ & $\begin{array}{c}\left\langle\mathcal{O}_{R}^{a b}\right\rangle \\
5.1 \times 10^{-20}\end{array}$ & $\begin{array}{c}\left\langle Q_{33}\right\rangle_{a b} \\
1.23 \times 10^{-19}\end{array}$ & $\begin{array}{c}\left\langle\hat{Q}_{33}\right\rangle_{a b} \\
9.4 \times 10^{-20}\end{array}$ & $\begin{array}{c}\left\langle\mathcal{O}_{I}^{a b}\right\rangle \\
2.4 \times 10^{-20}\end{array}$ \\
\hline
\end{tabular}

$$
\delta \operatorname{Re} d_{\tau}=\left(\sum_{a b} \frac{1}{\left(\delta \operatorname{Re} d_{\tau}\right)_{a b}^{2}}\right)^{-1 / 2}
$$

and analogously for $\delta \operatorname{Im} d_{\tau}$. Performing these quadratures with the uncertainties listed in Tables II, III, and IV yields the 1 s.d. errors $\delta \operatorname{Re} d_{\tau}$ and $\delta \operatorname{Im} d_{\tau}$ given in Table V. As to the optimal observables we assumed here for the purpose of comparison that they are measurable for all channels listed in Table IV. For the leptonic modes this may not be possible in an unambiguous way; see below. The numbers in Table $\mathrm{V}$ show that the sensitivity to $\operatorname{Re} d_{\tau}$ is improved by a factor of about 6 with the optimal observable $\mathcal{O}_{R}$ as compared to using the simple ones, whereas the sensitivity to $\operatorname{Im} d_{\tau}$ is improved by a factor of about 4 .

We briefly discuss the measurability of the observables used in this section. The KEKB accelerator is an asymmetric $e^{+} e^{-}$collider; particle momenta measured in the laboratory frame can of course be transformed to the $e^{+} e^{-}$ c.m. frame. The simple $C P$ observables (44)-(47) applied to the $\tau^{+} \tau^{-}$decay channels listed in Tables II, III require the momenta of charged mesons and of $e, \mu$ in the $e^{+} e^{-}$c.m. frame. They can be straightforwardly measured, except for the momentum of $\rho^{ \pm}$whose determination requires the reconstruction of the decay $\rho^{ \pm} \rightarrow \pi^{ \pm} \pi^{0}$.

The optimal observables involve the momenta of various particles from $\tau^{ \pm}$decay in the respective $\tau^{ \pm}$rest frame. This requires the knowledge of the $\tau^{ \pm}$momenta in the $e^{+} e^{-}$c.m. frame. If both $\tau^{+}$and $\tau^{-}$decay semihadronically their momenta can be reconstructed in an unambiguous way [36]. If one of the $\tau$ leptons decays semihadronically and the other one to either $e$ or $\mu$, one may discard radiative events in this class such that the $\tau^{+}$and $\tau^{-}$in the remaining events are, to good approximation, back to back and carry half of the c.m. energy in the $e^{+} e^{-}$frame. If the $\tau$ momentum can be reconstructed in the semihadronic decay, e.g., by reconstructing the $\tau$ production and decay vertices, the momentum of the leptonically decaying $\tau$ can be inferred. If both $\tau$ leptons decay leptonically the determination of their momenta is not possible in an unambiguous way. Therefore, we discard the results for the $\ell \ell^{\prime}$ channels in Table IV and add in quadrature the statistical errors of $\operatorname{Re} d_{\tau}$ and $\operatorname{Im} d_{\tau}$ attainable with the events listed in Table IV where both $\tau$ 's decay semihadronically and for the case where the semihadronic-leptonic decays of $\tau^{+} \tau^{-}$are added to the purely semihadronic events. The resulting 1 s.d. errors are given in Table VI. The numbers in this table and in Table $\mathrm{V}$ show that restriction to purely semihadronic $\tau^{+} \tau^{-}$decays does not lead to a significant decrease in sensitivity to $\operatorname{Re} d_{\tau}$ and $\operatorname{Im} d_{\tau}$.

Next we investigate the effects of cuts on the sensitivities to the $\tau$ EDM. A full-fledged Monte Carlo analysis with detailed cuts is beyond the scope of this paper. We analyze

TABLE VI. Ideal 1 s.d. statistical errors on $\operatorname{Re} d_{\tau}$ and $\operatorname{Im} d_{\tau}$ that result from adding in quadrature the respective uncertainties attainable with the optimal observables $\mathcal{O}_{R}^{a b}$ and $\mathcal{O}_{I}^{a b}$ in the semihadronic decays $(h h)$ and in the semihadronic and semihadronic-leptonic $(h h+h \ell)$ decays of $\tau^{+} \tau^{-}$.

\begin{tabular}{lcc}
\hline \hline & $\delta \operatorname{Re} d_{\tau}[e \mathrm{~cm}]$ & $\delta \operatorname{Im} d_{\tau}[e \mathrm{~cm}]$ \\
\hline$h h:$ & $5.8 \times 10^{-20}$ & $3.2 \times 10^{-20}$ \\
$h h+h \ell:$ & $5.1 \times 10^{-20}$ & $2.5 \times 10^{-20}$ \\
\hline \hline
\end{tabular}


TABLE VII. Optimal observables $\mathcal{O}_{R}^{a \bar{b}}$ and $\mathcal{O}_{I}^{a \bar{b}}$ at $\sqrt{s}=10.58 \mathrm{GeV}$ for the semihadronic $\tau$ decay channels with cuts specified in (96). In the case of nondiagonal channels the event numbers $N_{a b}$ include those of the charge-conjugate mode.

\begin{tabular}{lcccccc}
\hline \hline$\tau^{-} \rightarrow$ & $\tau^{+} \rightarrow$ & $N_{a b}$ & $w_{a \bar{b}}$ & $\delta \operatorname{Re} d_{\tau}\left(\times 10^{-19} e \mathrm{~cm}\right)$ & $\omega_{a \bar{b}}$ & $\delta \operatorname{Im} d_{\tau}\left(\times 10^{-19} e \mathrm{~cm}\right)$ \\
\hline$\pi^{-} \nu$ & $\pi^{+} \bar{\nu}$ & $4.21 \times 10^{8}$ & 0.128 & 2.54 & 0.359 & 1.52 \\
$\pi^{-} \pi^{0} \nu$ & $\pi^{+} \pi^{0} \bar{\nu}$ & $16.88 \times 10^{8}$ & 0.137 & 1.23 & 0.390 & 0.73 \\
$\pi^{-} \pi^{-} \pi^{+} \nu$ & $\pi^{+} \pi^{+} \pi^{-} \bar{\nu}$ & $1.73 \times 10^{8}$ & 0.139 & 3.81 & 0.408 & 2.22 \\
$\pi^{-} \nu$ & $\pi^{+} \pi^{0} \bar{\nu}$ & $16.53 \times 10^{8}$ & 0.135 & 1.25 & 0.386 & 0.74 \\
$\pi^{-} \nu$ & $\pi^{+} \pi^{+} \pi^{-} \bar{\nu}$ & $5.18 \times 10^{8}$ & 0.137 & 2.21 & 0.401 & 0.401 \\
$\pi^{-} \pi^{0} \nu$ & $\pi^{+} \pi^{+} \pi^{-} \bar{\nu}$ & $10.74 \times 10^{8}$ & 0.138 & 1.53 & 0.90 \\
\hline \hline
\end{tabular}

TABLE VIII. Ideal 1 s.d. statistical errors on $\operatorname{Re} d_{\tau}$ and $\operatorname{Im} d_{\tau}$ that result from adding in quadrature the respective uncertainties attainable with the optimal observables $\mathcal{O}_{R}^{a b}$ and $\mathcal{O}_{I}^{a b}$ in the semihadronic decays $(h h)$ of $\tau^{+} \tau^{-}$given in Table VII.

\begin{tabular}{lcc}
\hline \hline & $\delta \operatorname{Re} d_{\tau}[e \mathrm{~cm}]$ & $\delta \operatorname{Im} d_{\tau}[e \mathrm{~cm}]$ \\
\hline$h h:$ & $6.8 \times 10^{-20}$ & $4.0 \times 10^{-20}$ \\
\hline \hline
\end{tabular}

in the following only the expectation values of the optimal observables in the channels where both $\tau$ leptons decay semihadronically, as these observables and decay modes appear to have the highest sensitivity to $d_{\tau}$ and allow for an unambiguous reconstruction of the $\tau^{ \pm}$momenta. We apply the following $C P$-invariant phase-space cuts on the finalstate pions in the $e^{+} e^{-}$c.m. frame:

$$
23^{\circ}<\theta^{*}<157^{\circ}, \quad p_{T}>0.2 \mathrm{GeV},
$$

where $\theta^{*}$ is the polar angle of a pion with respect to the $e^{+} e^{-}$beam and $p_{T}$ its transverse momentum. ${ }^{5}$ Table VII contains the resulting coefficients $w_{a \bar{b}}$ and $\omega_{a \bar{b}}$ of the expectation values of $\mathcal{O}_{R}^{a \bar{b}}$ and $\mathcal{O}_{I}^{a \bar{b}}$, respectively, defined in (92). The event numbers and sensitivities given in Table VII are estimated by assuming an integrated luminosity of $50 \mathrm{ab}^{-1}$ that corresponds to assuming $N_{\tau \tau}=$ $4.5 \times 10^{10}$ in the case of no cuts. The expectation values are somewhat increased by the cuts while the event numbers are, of course, diminished. The resulting overall sensitivities are given in Table VIII. Comparing these numbers with those of Table VI shows that the cuts (96) lead only to a slight decrease in sensitivity to the $\tau$ EDM.

Moreover, the following remark is in order. As already indicated below Eq. (6) our results for the normalized expectation values listed in Tables II, III, IV, and VII do not depend on the fact that there is a resonance enhancement at $\sqrt{s}=10.58 \mathrm{GeV}$; these numbers hold also for the direct continuum production of $\tau$ pairs. In addition, we emphasize again that the event numbers, respectively, the integrated

\footnotetext{
${ }^{5}$ The cut on $\theta^{*}$ is inspired by the acceptance of the Belle II detector in the KEKB laboratory frame [19].
}

luminosity that we use for our sensitivity estimates to the $\tau$ EDM are expectations taken from [19].

The sensitivity to the $\tau$ EDM that the Belle II experiment may eventually achieve with purely semihadronic $\tau^{+} \tau^{-}$ decays was investigated also in [16]. The authors of this paper use the term proportional to $d_{\tau}$ of the matrix element for $e^{+} e^{-} \rightarrow \tau^{+} \tau^{-} \rightarrow h \nu_{\tau} h^{\prime} \bar{\nu}_{\tau}$ as optimal observable. It is evaluated with the momenta of the mesons and the reconstructed one of the neutrinos. The real and imaginary parts of the $\tau$ EDM are not separately determined. Assuming the same $\tau^{+} \tau^{-}$event number as we did above, the authors of Ref. [16] find that a 1 s.d. statistical sensitivity $\delta\left|d_{\tau}\right|=2 \times 10^{-19} e \mathrm{~cm}$ can be achieved with their approach.

\section{THE $\tau$ EDM FORM FACTOR IN SOME SM EXTENSIONS}

In the SM the EDM $d_{\ell}$ of a charged lepton is extremely tiny and generated only at high loop order. The dominant shortdistance contribution to $d_{\ell}$ is thought to arise via KobayashiMaskawa phase induced four-loop contributions that contain, for instance, the induced EDM form factor of the $W$ boson. It can be estimated to be of the order $d_{\tau} \sim \mathcal{O}\left(10^{-42}\right) e \mathrm{~cm}$. (One may take, for instance, the estimate of [37] for $d_{e}$ and apply it to the $\tau$ lepton.) Recently it was pointed out that long-distance hadronic contributions are considerably larger [38]. For the $\tau$ EDM was found that these contributions amount to $d_{\tau} \simeq$ $-7.3 \times 10^{-38} e \mathrm{~cm}$ [38]. Nevertheless, this is undetectable for the time being.

Thus, the detection of a nonzero particle EDM, in particular of the $\tau$ lepton, in a present-day experiment or one in the foreseeable future would be evidence for a new type of $C P$ violation. In this section we consider three SM extensions with $\mathrm{CP}$-violating interactions that generate EDM form factors of fundamental fermions already at one loop. The models we are interested in have $C P$ violating Yukawa couplings. These interactions can induce a $\tau$ EDM that can be much larger than the electron EDM generated in these models. ${ }^{6}$ We compute the $\tau$ EDM at one loop in a type-II two-Higgs-doublet model and in two

\footnotetext{
${ }^{6} \mathrm{We}$ recall that in models with Higgs-Yukawa-like $C P$-violating couplings the dominant contribution to the electron EDM occurs at two loops [39].
} 
scalar leptoquark models and investigate its potential magnitude in the timelike region $q^{2} \sim(10 \mathrm{GeV})^{2}$, taking into account phenomenological constraints, in particular the tight upper bound (1) on the electron EDM.

\section{A. Type-II two-Higgs doublet extension}

In two-Higgs doublet models (2HDM) the field content of the SM is extended by an additional Higgs doublet $\mathrm{H}_{2}$. We consider here as an example the so-called type-II model. It is defined by its Yukawa coupling structure: the doublet $H_{1}$ is coupled to right-chiral down-type quarks and charged leptons, while $H_{2}$ is coupled to right-chiral uptype quarks only. By construction, flavor-changing neutral currents are absent at tree level in this model. Assuming a $C P$-violating Higgs potential $V\left(H_{1}, H_{2}\right)$ the particle spectrum of the $2 \mathrm{HDM}$ contains three neutral Higgs bosons $h_{j}$ $(j=1,2,3)$ that are $C P$ mixtures. In flavor-conserving 2HDM their Yukawa couplings to quarks and leptons are of the form

$$
\mathcal{L}_{Y, f}=-\left(\sqrt{2} G_{F}\right)^{1 / 2} m_{f}\left[a_{f, j} \bar{f} f-b_{f, j} \bar{f} i \gamma_{5} f\right] h_{j},
$$

where $f=q, \ell, G_{F}$ is the Fermi constant, and the reduced Yukawa couplings $a_{f, j}$ and $b_{f, j}$ depend on the specific type of 2HDM. In the type-II model the reduced couplings of the mass eigenstates $h_{j}$ to the $\tau$ lepton are (we use here the conventions of [40])

$$
a_{\tau, j}=R_{j 1} / \cos \beta, \quad b_{\tau, j}=R_{j 3} \tan \beta .
$$

Here $\tan \beta=v_{2} / v_{1}$ is the ratio of the vacuum expectation values of the two Higgs doublet fields, and $\left(R_{i j}\right)$ is a real orthogonal matrix that relates the $C P$ eigenstates and the mass eigenstates of the three physical neutral Higgs bosons. The relations (98) hold also for the other charged leptons and the down-type quarks. (For up-type quarks, see for instance [40].) If $a_{f, j} b_{f, j} \neq 0$, then (97) violates $C P$.

Here we identify $h_{1}$ with the $125 \mathrm{GeV}$ Higgs boson and assume that $h_{2}$ and $h_{3}$ are heavier than $400 \mathrm{GeV}$. The exchange of the $h_{j}$ induces a $\tau$ EDM at one loop shown by the diagram Fig. 3(a). With the convention of Eq. (7) we get $^{7}$

$$
\begin{gathered}
d_{\tau}(s)=\sum_{j=1}^{3} a_{\tau, j} b_{\tau, j} d_{\tau}^{(j)}(s) \\
d_{\tau}^{(j)}(s)=-\frac{e \sqrt{2} G_{F} m_{\tau}^{3}}{4 \pi^{2} s \beta_{\tau}^{2}}\left[B_{0}\left(s, m_{\tau}^{2}, m_{\tau}^{2}\right)-B_{0}\left(m_{\tau}^{2}, m_{j}^{2}, m_{\tau}^{2}\right)\right. \\
\left.+m_{j}^{2} C_{0}\left(s, m_{\tau}^{2}, m_{j}^{2}, m_{\tau}^{2}\right)\right]
\end{gathered}
$$

\footnotetext{
${ }^{7}$ The real and imaginary parts of the EDM form factor of a fermion were computed for a class of 2HDM including the type-II model in [41] and evaluated for the top quark.
}



(a)

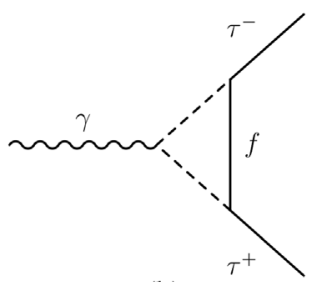

(b)
FIG. 3. One-loop diagrams that contribute to the $\tau$ EDM form factor in the models considered in Sec. VI. In the type-II 2HDM only diagram (a) contributes and the dashed and solid internal lines correspond to $h_{j}(j=1,2,3)$ and $\tau$, respectively. In the leptoquark models both diagrams contribute and the dashed and solid internal lines correspond to a spin-zero leptoquark and the top quark, respectively.

where $\beta_{\tau}=\left(1-4 m_{\tau}^{2} / s\right)^{1 / 2}$ and $m_{j}$ is the mass of $h_{j}$. The functions $B_{0}$ and $C_{0}$ denote the standard scalar one-loop two-point and three-point functions [42]. For $s \geq 4 m_{\tau}^{2}$ the EDM form factor (99) has both a real and an imaginary part.

However, apart from the upper bound (1) on the electron EDM existing constraints from experiments at the LHC preclude a $\tau$ EDM of order $10^{-20} e \mathrm{~cm}$ or larger in this model. A recent analysis of the decay of the $125 \mathrm{GeV}$ Higgs boson to $\tau^{+} \tau^{-}$by the CMS experiment restricts the size of a potentially existing pseudoscalar coupling of $h_{1}$ to the $\tau$ lepton: $\left|b_{\tau, 1} / a_{\tau, 1}\right| \leq 0.38$ at $68 \%$ C.L. [43]. Searches for additional neutral Higgs bosons with decays to $\tau^{+} \tau^{-}$ exclude Higgs-boson masses of about $400 \mathrm{GeV}$ and below for a large range of Higgs coupling to $\tau$ leptons; see, for example, [44,45] and references therein.

We exemplify the order of magnitude of $d_{\tau}$ that is compatible with these constraints by assuming the masses of the Higgs bosons $h_{2}$ and $h_{3}$ to be $m_{2}=500 \mathrm{GeV}$ and $m_{3}=800 \mathrm{GeV}$, respectively. Moreover, we choose $\tan \beta=$ 1 and the angles of the mixing matrix $R$, in the parametrization of [40], to be $\alpha_{1}=\alpha_{3}=0.785, \alpha_{2}=0.209$. The resulting real and imaginary parts of the $\tau$ EDM (99) are given in Table IX for several c.m. energies in the energy range considered in this paper.

By and large the order of magnitude of the $\tau$ EDM form factor listed in Table IX is characteristic for a large class of Higgs models. Significantly larger values of $\operatorname{Re} d_{\tau}(s)$ and $\operatorname{Im} d_{\tau}(s)$ would be possible if, for instance, Higgs bosons exist with exclusive $C P$-violating couplings to the third

TABLE IX. Values of the real and imaginary parts of the $\tau$ EDM form factor (99) in the type-II 2HDM, evaluated with the parameter choice given in the text.

\begin{tabular}{lcccc}
\hline \hline$\sqrt{s}[\mathrm{GeV}]$ & 3.6 & 4 & 10.58 & 12 \\
\hline $\operatorname{Re} d_{\tau}(s)\left[10^{-24} e \mathrm{~cm}\right]$ & 2.24 & 2.13 & 1.38 & 1.30 \\
$\operatorname{Im} d_{\tau}(s)\left[10^{-24} e \mathrm{~cm}\right]$ & 0.13 & 0.38 & 0.77 & 0.78 \\
\hline \hline
\end{tabular}


generation of quarks and leptons, such that the stringent constraint (1) on the electron EDM can be evaded.

\section{B. Spin-zero leptoquarks}

Leptoquarks, whose interactions connect a lepton and a quark, occur naturally in unified models of strong and electroweak interactions. In recent years they have come again into the focus of numerous investigations in the context of possible explanations of semileptonic $B$ and $D$ meson decay and muon $(g-2)$ anomalies; see, for instance, [46-50] and references therein. Here we are interested in spin-zero leptoquarks with $C P$-violating Yukawa couplings. They can generate EDMs of the muon and tau lepton that are significantly larger than that of the electron, ${ }^{8}$ as pointed out some time ago in [24,52] (cf. also [53]).

We consider in the following two different spin-zero leptoquark models, namely the SM extended by a weak $\mathrm{SU}(2)$ leptoquark doublet $\Phi$ with $\mathrm{SU}(3)_{\mathrm{c}} \times \mathrm{SU}(2)_{\mathrm{L}} \times \mathrm{U}_{\mathrm{Y}}(1)$ quantum numbers $\Phi(3,2,7 / 6)$ (model I) and a $\mathrm{SM}$ extension by a weak singlet $S$ with quantum numbers $S(3,1,-1 / 3)$ (model II). The gauge-invariant interaction Lagrangians are [54]

$$
\begin{aligned}
& \mathcal{L}_{I}=\left[\overline{L_{L}} \Lambda_{L} \epsilon u_{R}+\overline{e_{R}} \Lambda_{R} Q_{L}\right] \Phi^{\dagger}+\text { H.c. }, \\
& \mathcal{L}_{I I}=\left[\overline{L_{L}^{c}} Y_{L} \epsilon Q_{L}+\overline{e_{R}^{c}} Y_{R} u_{R}\right] S^{\dagger}+\text { H.c. }
\end{aligned}
$$

Here $L_{L}=\left(\nu_{i L}, e_{i, L}\right)^{T}, \quad Q_{L}=\left(u_{i, L}, d_{i, L}\right)^{T}, \quad e_{R}=\left(e_{i, R}\right)$, $u_{R}=\left(u_{i, R}\right)$, where $i=1,2,3$ is a generation index. The label $c$ denotes charge conjugation. The $2 \times 2$ matrix $\epsilon=$ $i \tau_{2}$ acts on the $\mathrm{SU}(2)$ indices. The electric charge (in units of $e>0$ ) of $S$ is $Q_{S}=-1 / 3$. For the components of the doublet $\Phi=\left(\varphi, \varphi^{\prime}\right)^{T}$ we have $Q_{\varphi}=5 / 3$ and $Q_{\varphi^{\prime}}=2 / 3$. The $\Lambda_{L}, \Lambda_{R}$ and $Y_{L}, Y_{R}$ denote complex $3 \times 3$ matrices in flavor space. Usually the interactions (101) and (102) are defined in the weak basis and are rotated, after electroweak symmetry breaking, to the mass basis. We can choose a basis in which the Yukawa matrices of the up-type quark and of the charged-lepton couplings to the SM Higgs boson are already diagonal. Then only the down-type quark and neutrino fields must be rotated with their respective mixing matrices when one transforms to the mass basis. The interactions in (101) involving charged leptons and uptype quarks, with which we are concerned here, remain unaffected.

We assume that the off-diagonal elements of the matrices $\Lambda_{L}, \Lambda_{R}$ and $Y_{L}, Y_{R}$ in generation space are very small and can be neglected. Let us denote

$\lambda_{J}=\left(\Lambda_{J}\right)_{33} \quad$ and $\quad y_{J}=\left(Y_{J}\right)_{33}, \quad J=L, R$,

and

\footnotetext{
${ }^{8}$ A recent analysis of the effects of spin-zero leptoquarks on the EDMs of leptons, quarks, and nucleons was made in [51].
}

$$
f_{\mathrm{I}}=\operatorname{Im}\left(\lambda_{L}^{*} \lambda_{R}\right) \quad \text { and } \quad f_{\mathrm{II}}=\operatorname{Im}\left(y_{R}^{*} y_{L}\right) .
$$

If $f_{\mathrm{I}} \neq 0\left[f_{\mathrm{II}} \neq 0\right]$, then the interaction Eq. (101) [Eq. (102)] generates a nonzero $\tau$ EDM at one loop. It is represented by Figs. 3(a) and 3(b) where the internal fermion and boson lines correspond to the top quark $t$ and the $\varphi$ leptoquark in model I and to $t$ and $S$ in model II, respectively. The $\tau$ EDM form factor is given by [24]

$d_{\tau}(s)=e m_{t} N_{c} \frac{f_{\kappa}}{8 \pi^{2}} \frac{1}{s \beta_{\tau}^{2}}\left[Q_{t} K_{t}(s)-Q_{\chi} K_{\chi}(s)\right], \quad \kappa=\mathrm{I}, \mathrm{II}$,

where $N_{c}=3, m_{t}$ is the mass of the top quark which provides the chirality flip, $Q_{t}=2 / 3$ and $Q_{\chi}=5 / 3(-1 / 3)$ in case of model I (II), where $\chi$ denotes either $\varphi$ or $S$. Moreover,

$$
\begin{aligned}
K_{t}(s)= & B_{0}\left(s, m_{t}^{2}, m_{t}^{2}\right)-B_{0}\left(m_{\tau}^{2}, m_{t}^{2}, m_{\chi}^{2}\right) \\
& +\left(m_{\chi}^{2}+m_{\tau}^{2}-m_{t}^{2}\right) C_{0}\left(s, m_{t}^{2}, m_{\chi}^{2}, m_{t}^{2}\right), \\
K_{\chi}(s)= & B_{0}\left(s, m_{\chi}^{2}, m_{\chi}^{2}\right)-B_{0}\left(m_{\tau}^{2}, m_{t}^{2}, m_{\chi}^{2}\right) \\
+ & \left(s / 2+m_{t}^{2}-m_{\chi}^{2}-m_{\tau}^{2}\right) C_{0}\left(s, m_{\chi}^{2}, m_{t}^{2}, m_{\chi}^{2}\right) .
\end{aligned}
$$

Here $m_{\chi}$ is the mass of $\varphi(S)$ in the case of model I (II). Because $m_{t}, m_{\varphi}, m_{S} \gg \sqrt{s}$ in the kinematic range that we consider here, the $\tau$ EDM form factor (105) is real.

In order to estimate the potential size of $d_{\tau}$ we choose the leptoquark masses $m_{\chi}=1.5 \mathrm{TeV}(\chi=\varphi, S)$ which are compatible with the experimental bounds from LHC $[55,56]$ and the constraints from the anomalous magnetic moments of the electron and muon [49]. For comparison we evaluate (105) also for $m_{\chi}=1 \mathrm{TeV}$ and $2 \mathrm{TeV}$. With $m_{t}=172.4 \mathrm{GeV}$ [1] we get from (105) the values listed in Table X.

The numbers in Table $\mathrm{X}$ show that for a given leptoquark mass the form factor $\operatorname{Re} d_{\tau}(s)$ is essentially flat in the kinematic range considered here. So far, the experimental

TABLE X. Values of the $\tau$ EDM form factor (105) in the doublet (I) and singlet (II) leptoquark model. The numbers in the first, second, and third rows of each model are obtained with $m_{\chi}=1,1.5$, and $2 \mathrm{TeV}(\chi=\varphi, S)$. Moreover, we use $m_{t}=172.4 \mathrm{GeV}$.

\begin{tabular}{lrrrr}
\hline \hline$\sqrt{s}[\mathrm{GeV}]$ & 3.6 & \multicolumn{1}{c}{4} & 10.58 & 12 \\
\hline Model I: $\operatorname{Re} d_{\tau}(s)\left[10^{-20} f_{\mathrm{I}} e \mathrm{~cm}\right]$ & 14.44 & 14.44 & 14.45 & 14.45 \\
& 7.89 & 7.89 & 7.89 & 7.89 \\
& 5.04 & 5.04 & 5.04 & 5.04 \\
Model II: $\operatorname{Re} d_{\tau}(s)\left[10^{-20} f_{\mathrm{II}} e \mathrm{~cm}\right]$ & 8.85 & 8.85 & 8.86 & 8.86 \\
& 5.24 & 5.24 & 5.25 & 5.25 \\
& 3.51 & 3.51 & 3.51 & 3.51 \\
\hline \hline
\end{tabular}


bounds on the $C P$ parameters $f_{\mathrm{I}}, f_{\mathrm{II}}$ are not stringent. Using the experimental bound (3) and the numbers given in Table $\mathrm{X}$ for $\sqrt{s}=10.58 \mathrm{GeV}$ and $m_{\chi}=1.5 \mathrm{TeV}$, we get

$$
\left|f_{\mathrm{I}}\right|<570, \quad\left|f_{\mathrm{II}}\right|<857 \quad \text { for } m_{\chi}=1.5 \mathrm{TeV} .
$$

If leptoquark couplings to the $\tau$ lepton and the $c$ quark are taken into account in (101) and (102), then $d_{\tau}(s)$ develops also an imaginary part for $\sqrt{s}>2 m_{c}$. However, away from the charm threshold, the $c$-quark contribution to $d_{\tau}$ is suppressed in magnitude by the factor $m_{c} / m_{t} \sim 10^{-2}$ as compared to the leading contribution (105), regardless of additional suppression due to small off-diagonal Yukawa couplings.

One may expect that the Yukawa couplings of the spinzero leptoquarks are of the Higgs-boson type. Then the (diagonal) couplings of $\varphi$ and $S$ in (101) and (102) will be proportional to the right-handed fermion involved. That is,

$$
\begin{aligned}
\lambda_{L} & \sim m_{t} / M_{\mathrm{I}}, & & \lambda_{R} \sim m_{\tau} / M_{\mathrm{I}}, \\
y_{L} & \sim m_{\tau} / M_{\mathrm{II}}, & y_{R} & \sim m_{t} / M_{\mathrm{II}},
\end{aligned}
$$

where $M_{\mathrm{I}}$ and $M_{\mathrm{II}}$ are mass scales that are expected to be larger than the electroweak symmetry breaking scale $v=246 \mathrm{GeV}$. In this case the magnitude of $\operatorname{Re} d_{\tau}(s)$ will be smaller by a factor of at least $10^{-2}$ than the numbers listed in Table X.

\section{Box contributions}

The one-loop $S$-matrix element of $e^{+} e^{-} \rightarrow \tau^{+} \tau^{-}$can receive in SM extensions also one-particle irreducible $C P$-violating box contributions that involve Lorentz structures such as $(\bar{e} e)\left(\bar{\tau} i \gamma_{5} \tau\right)$. Here we argue that in the models considered in Secs. VI A and VI B these contributions that are depicted in Fig. 4 can be neglected compared to those of the $\tau$ EDM form factors.

In the type-II 2HDM only diagram Fig. 4(a) appears. From the Yukawa interaction (97) one obtains that this



(a)

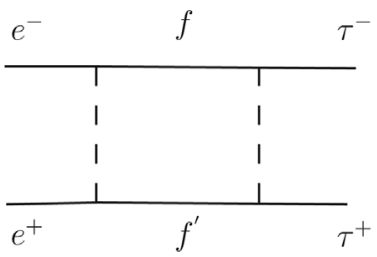

(b)
FIG. 4. One-loop box diagrams that contribute to the $S$-matrix element of $e^{+} e^{-} \rightarrow \tau^{+} \tau^{-}$in the models considered in Secs. VI A and VIB. In the type-II 2HDM only diagram (a) contributes and the dashed and solid internal lines correspond to $h_{j}(j=1,2,3)$ and $f=e, f^{\prime}=\tau$, respectively. In the leptoquark models both diagrams can contribute and the dashed and solid internal lines correspond to a spin-zero leptoquark and an up-type quark, respectively. Crossed diagrams are not shown. contribution is proportional to $G_{F} m_{e}^{2}$. Thus this contribution to the $S$-matrix element of $e^{+} e^{-} \rightarrow \tau^{+} \tau^{-}$is negligible compared to that of the $\tau$ EDM form factor (100).

As to the spin-zero leptoquark models: If one considers interactions (101), (102) that are diagonal in generation space, then only diagram (a) contributes with $f=u, f^{\prime}=t$, and this contribution entails a suppression factor $m_{u} / \sqrt{s}$ where $m_{u}$ is the mass of the $u$ quark. In the case of interactions that are nondiagonal in generation space, diagrams (a) and (b) contribute, but those contributions that involve leptoquark couplings between the electron and the $c$ and $t$ quark contain off-diagonal matrix elements $\left(\Lambda_{J}\right)_{1 j}$ or $\left(Y_{J}\right)_{1 j}(J=L, R, j \neq 1)$ that are small due to experimental constraints (see, e.g., [49,50]).

Thus we conclude that within the above SM extensions the $C P$-violating part of the one-loop $S$-matrix element of $e^{+} e^{-} \rightarrow \tau^{+} \tau^{-}$is given to very good approximation by the contribution from the $\tau$ EDM form factor. In addition, we remark that the one-loop EDM form factors computed in Secs. VI A and VI B are gauge invariant. Needless to say, the contribution of the electron EDM form factor to this matrix element is completely irrelevant.

\section{CONCLUSIONS}

The huge data samples of $\tau^{+} \tau^{-}$production and decay that will eventually be recorded at existing low-energy $e^{+} e^{-}$colliders will allow, among other investigations, the search for a $\tau$ electric dipole form factor $d_{\tau}(s)$ with a precision that is significantly higher than existing bounds. We reconsidered the issue of using simple and optimal $C P$ observables for such measurements. We discussed the general formalism of optimal observables and applied it to two $C P$-odd observables based on $C P$-odd $\tau$-spin correlations and polarization asymmetries that are sensitive to the real and imaginary parts of $d_{\tau}(s)$, respectively. Special emphasis was put on the covariance of these observables. In our numerical analysis we computed the expectation values and covariances of the optimal $C P$ observables for $\tau$-pair production in $e^{+} e^{-}$collisions at the $\Upsilon(4 \mathrm{~S})$ resonance with subsequent decays of $\tau^{ \pm}$to major leptonic or semihadronic modes. These results hold also for the continuum production of $\tau$ pairs at $\sqrt{s}=10.58 \mathrm{GeV}$. For the $\tau$ decays to two pions and three charged pions we took the full kinematic information of the hadronic system into account by incorporating the respective differential $\tau^{ \pm}$ decay density matrices into the optimal observables. In this way the maximal $\tau$-spin analyzing power is obtained also with these decay modes. Assuming that the Belle II experiment will eventually record and analyze $4.5 \times 10^{10}$ $\tau^{+} \tau^{-}$events at $\sqrt{s}=10.58 \mathrm{GeV}$ we found that with purely semihadronic $\tau^{+} \tau^{-}$decays 1 s.d. sensitivities $\delta \operatorname{Re} d_{\tau}=$ $5.8 \times 10^{-20} e \mathrm{~cm}$ and $\delta \operatorname{Im} d_{\tau}=3.2 \times 10^{-20} e \mathrm{~cm}$ can be obtained with these optimal observables. For $\operatorname{Re} d_{\tau}$ this is better than a factor of 5 and for $\operatorname{Im} d_{\tau}$ better than a factor of 3 
as the sensitivities attainable with the simple $C P$-odd observables that we analyzed, too. Including events where one (or both) $\tau$ leptons decay leptonically does not lead to a significant increase in sensitivity to $\operatorname{Re} d_{\tau}$ and $\operatorname{Im} d_{\tau}$. These results were obtained without cuts. We analyzed also the sensitivity of the optimal observables to $\operatorname{Re} d_{\tau}$ and $\operatorname{Im} d_{\tau}$ in the purely semihadronic $\tau^{+} \tau^{-}$decay channels by applying cuts on the final-state pions. Assuming an integrated luminosity of $50 \mathrm{ab}^{-1}$, which corresponds to the above number of $\tau^{+} \tau^{-}$events in the case of no cuts, we obtained $\delta \operatorname{Re} d_{\tau}=6.8 \times 10^{-20} e \mathrm{~cm}$ and $\delta \operatorname{Im} d_{\tau}=4.0 \times 10^{-20} e \mathrm{~cm}$. That is, the 1 s.d. sensitivities decrease only slightly.

Furthermore, we discussed a few SM extensions with nonstandard $C P$ violation that predict a nonzero $\tau$ EDM already at one-loop order. The tight experimental upper bound on the electron EDM, experimental results from the LHC on the $C P$ nature of the $125 \mathrm{GeV}$ Higgs boson, and bounds on the mass and couplings of new particles severely constrain the potential magnitude of $d_{\tau}$. Within the type-II 2HDM, which we consider in this context to be exemplary for a large class of two-Higgs doublet extensions of the SM, the $\tau$ EDM form factor turns out to be too small to be detected in the foreseeable future. However, in scalar leptoquark extensions of the $\operatorname{SM~} \operatorname{Re} d_{\tau}(s) \sim 10^{-20} e \mathrm{~cm}$ is still possible in the energy range considered in this paper. In any case, future $\tau$ EDM measurements with the Belle II and also the BES III experiment using optimal observables will provide significant information about new sources of $C P$ violation.

\section{ACKNOWLEDGMENTS}

The authors thank M. Diehl, R. Karl, F. M. Krinner, F. Nerling, A. Rostomyan, and A. Szczurek for discussions and correspondence, and C. Ewerz for help with one figure. The work of L.C. was supported by the Deutsche Forschungsgemeinschaft under Grant No. 396021762TRR 257.

\section{APPENDIX A: $\tau$ DECAY DENSITY MATRICES}

Here we list the density matrices that describe several major decays of polarized $\tau^{\mp}$. Most of them given below are used in Sec. V. The kinematic variables in this appendix are defined in the respective $\tau^{ \pm}$rest frame unless stated otherwise. The decay density matrices are computed in the Standard Model; potential $C P$-violating effects in $\tau$ decays are not taken into account.

First we consider $\tau^{\mp}$ decays into one charged prong, $\tau^{\mp} \rightarrow a^{\mp}+X$ with particle multiplicity $\left\langle n_{a}\right\rangle=1$. The charged particle $a^{\mp}$ acts as the $\tau^{\mp}$ spin analyzer. Assuming $C P$ invariance in the decays of $\tau^{\mp}$ we have in the $\tau$ rest frame

$$
\left\langle a^{-}(\mathbf{q}), X|\mathcal{T}| \tau_{\beta}^{-}\right\rangle=\eta_{a}\left\langle a^{+}(-\mathbf{q}), X^{C P}|\mathcal{T}| \tau_{\beta}^{+}\right\rangle,
$$

where $X^{C P}$ is the $C P$ transform of $X$ and $\eta_{a}= \pm 1$. If $a$ denotes a lepton, we have $\eta_{a}=1$; for $a=$ meson, $\eta_{a}$ is the product of the intrinsic parity and charge-parity quantum numbers of $a$. Thus, for a pion ( $\rho$ meson) we get $\eta_{\pi}=-1$ $\left(\eta_{\rho}=+1\right)$. Equation (A1) implies for the $\tau^{\mp}$ decay density matrices

$$
\mathcal{D}_{\beta^{\prime} \beta}^{a^{-}}\left(\tau^{-} \rightarrow a^{-}(\mathbf{q})+X\right)=\mathcal{D}_{\beta^{\prime} \beta}^{a^{+}}\left(\tau^{+} \rightarrow a^{+}(-\mathbf{q})+X^{C P}\right) .
$$

The respective decay density matrix $\mathcal{D}^{a}=\left(\mathcal{D}_{\beta^{\prime} \beta}^{a}\right)$ defined in (26) and (27) is of the form

$$
\begin{aligned}
& \frac{d^{3} q_{\mp}}{(2 \pi)^{3} 2 E_{a^{\mp}}} \mathcal{D}^{a^{\mp}}\left(\tau^{\mp} \rightarrow a^{\mp}\left(q_{\mp}\right)+X\right) \\
& =d E_{a^{\mp}} \frac{d \Omega_{a^{\mp}}}{4 \pi} n\left(E_{a^{\mp}}\right)\left[\mathbb{1} \pm h\left(E_{a^{\mp}}\right) \hat{\mathbf{q}}_{\mp} \cdot \boldsymbol{\sigma}\right],
\end{aligned}
$$

where $E_{a^{\mp}}$ is the energy of $a^{\mp}$ and $d \Omega_{a^{\mp}}=d \cos \theta_{a^{\mp}} d \varphi_{a^{\mp}}$. In (A3) the symbol $\mathbb{1}$ denotes the two-dimensional unit matrix and $\boldsymbol{\sigma}=\left(\sigma_{1}, \sigma_{2}, \sigma_{3}\right)$ is the vector of Pauli matrices. The function $n\left(E_{a}\right)$ determines the energy spectrum of $\tau \rightarrow a$ while $h\left(E_{a}\right)$ encodes the $\tau$-spin analyzing power of the charged prong. Equation (A3) is used in the calculations of Sec. V. If the right-hand side of (A3) is integrated over $E_{a^{\mp}}$, it takes, due to the normalization convention (27), the form

$$
\begin{aligned}
& \frac{d \boldsymbol{\Omega}_{a^{\mp}}}{4 \pi} \int d E_{a^{\mp}} n\left(E_{a^{\mp}}\right)\left[\mathbb{1} \pm h\left(E_{a^{\mp}}\right) \hat{\mathbf{q}}_{\mp} \cdot \boldsymbol{\sigma}\right] \\
& \quad=\frac{d \boldsymbol{\Omega}_{a^{\mp}}}{4 \pi}\left[\mathbb{1} \pm \alpha_{a} \hat{\mathbf{q}}_{\mp} \cdot \boldsymbol{\sigma}\right],
\end{aligned}
$$

where $\alpha_{a}\left(\left|\alpha_{a}\right| \leq 1\right)$ is a measure of the $\tau$ spin-analyzing power of $a$.

Next we list the spectral functions $n\left(E_{a}\right)$ and $h\left(E_{a}\right)$ of several decay density matrices (A3). The functions $n\left(E_{a}\right)$ have dimension 1/energy while the functions $h\left(E_{a}\right)$ are dimensionless.

\section{The decay $\tau^{\mp} \rightarrow \ell^{\mp}\left(q_{\mp}\right)+\nu_{\ell} \nu_{\tau}$}

In the leptonic decays $\tau^{\mp} \rightarrow \ell^{\mp} \nu_{\ell} \nu_{\tau}$ the mass of $\ell=e, \mu$ can be neglected. (Here and below the symbol $\nu$ denotes a neutrino or antineutrino, depending on the case.) Using $x=2 E_{\ell} / m_{\tau}$, where $E_{\ell}$ is defined in the $\tau$ rest frame, one has [57]

$$
n_{\ell}\left(E_{\ell}\right)=\frac{4}{m_{\tau}} x^{2}(3-2 x), \quad h_{\ell}\left(E_{\ell}\right)=\frac{1-2 x}{3-2 x}
$$

with $0 \leq x \leq 1$. Integrating over the charged lepton energy in (A3) yields (A4) with the $\tau$-spin analyzing power 


$$
\alpha_{\ell}=-\frac{1}{3}
$$

The value of $\alpha_{\ell}$ can be increased by a suitable cut on $E_{\ell}$.

$$
\text { 2. The decay } \tau^{\mp} \rightarrow \pi^{\mp}\left(q_{\mp}\right)+\nu_{\tau}
$$

In the two-body decay $\tau \rightarrow \pi+\nu_{\tau}$ the energy $E_{\pi}$ in the $\tau$ rest frame is fixed and the functions $n_{\pi}\left(E_{\pi}\right)$ and $h_{\pi}\left(E_{\pi}\right)$ are given by [57]:

$$
n_{\pi}\left(E_{\pi}\right)=\delta\left(E_{\pi}-\frac{m_{\tau}^{2}+m_{\pi}^{2}}{2 m_{\tau}}\right), \quad h_{\pi}\left(E_{\pi}\right)=1
$$

Here the $\tau$-spin analyzing power is maximal,

$$
\alpha_{\pi}=1
$$

\section{The decay $\tau^{\mp} \rightarrow \rho^{\mp}\left(q_{\mp}\right)+\nu_{\tau}$}

If the four-momentum of the intermediate $\rho$ meson can be determined in the decay $\tau^{\mp} \rightarrow \pi^{\mp} \pi^{0} \nu_{\tau}$ by measuring the energies and momenta of both $\pi^{\mp}$ and $\pi^{0}$, the $\rho$ meson can be used as a $\tau$-spin analyzer. It is well known that in the two-body decay of a polarized $\tau$ to a transversely or longitudinally polarized spin- 1 meson and $\nu_{\tau}$ the $\tau$-spin analyzing power of the meson is maximal [58]. However, the polarization of the vector meson cannot be determined event by event. Summing over the polarizations of the $\rho$ meson and treating it as an on-shell particle, one obtains $\tau^{\mp}$ decay density matrices of the form (A3) with the spectral functions $[57,58]$

$n_{\rho}\left(E_{\rho}\right)=\delta\left(E_{\rho}-\frac{m_{\tau}^{2}+m_{\rho}^{2}}{2 m_{\tau}}\right), \quad h_{\rho}\left(E_{\rho}\right)=\frac{m_{\tau}^{2}-2 m_{\rho}^{2}}{m_{\tau}^{2}+2 m_{\rho}^{2}}$

Using $m_{\rho}=0.775 \mathrm{GeV}$ we obtain

$$
\alpha_{\rho}=0.45
$$

We use this two-body decay mode with (A3) and (A9) in our analysis of the simple $C P$ observables in Sec. V.

\section{The decay $\tau^{\mp} \rightarrow \pi^{\mp}\left(q_{1}\right)+\pi^{0}\left(q_{2}\right)+\nu_{\tau}$}

The differential rate of the decay of polarized $\tau$ leptons to a charged and neutral pion via a $\rho$ meson was calculated in [57] in the on-shell approximation for the intermediate $\rho$ meson. A more elaborate description of this decay mode takes the $\rho$ and $\rho^{\prime}$ resonances and their finite widths as intermediate states into account [59-61]. We use the matrix element of $[59,60]$ for the decay chain $\tau \rightarrow \rho\left(\rho^{\prime}\right) \rightarrow 2 \pi \nu_{\tau}$. Exact isospin invariance is assumed. In the $\tau^{-}$rest frame we obtain for the $\tau^{-} \rightarrow \pi^{-} \pi^{0} \nu_{\tau}$ decay density matrix $\mathcal{D}^{2 \pi}$ that is differential in the pion momenta:

$$
\begin{aligned}
& \prod_{i=1}^{2} \frac{d^{3} q_{i}}{(2 \pi)^{3} 2 q_{i}^{0}} \mathcal{D}^{2 \pi}\left(\tau^{-}(k) \rightarrow \pi^{-}\left(q_{1}\right) \pi^{0}\left(q_{2}\right) \nu_{\tau}\right) \\
& \quad=\frac{1}{2 m_{\tau} \Gamma_{2 \pi}} d \Phi_{2}\left|\mathcal{M}_{2}\right|^{2},
\end{aligned}
$$

where $\Gamma_{2 \pi}=\Gamma\left(\tau^{-} \rightarrow \pi^{-} \pi^{0} \nu_{\tau}\right)$ and

$$
\left|\mathcal{M}_{2}\right|^{2}=G_{F}^{2}\left|V_{u d}\right|^{2}\left|F_{\pi}\left(Q^{2}\right)\right|^{2}\left(A_{2} \rrbracket+\boldsymbol{H}_{2} \cdot \boldsymbol{\sigma}\right) .
$$

Here $G_{F}$ and $V_{u d}$ denote the Fermi constant and the $u d$ Cabibbo-Kobayashi-Maskawa matrix element, respectively. The terms in the squared matrix element are

$$
\begin{gathered}
A_{2}=4\left[2(k \cdot q)^{2}+q^{2}\left(Q^{2}-k \cdot Q\right)\right], \\
H_{2}^{j}=4 m_{\tau}\left[2(k \cdot q) q^{j}+q^{2} Q^{j}\right],
\end{gathered}
$$

where $j=1,2,3, k=\left(m_{\tau}, \mathbf{0}\right)^{T}$ in the $\tau$ rest frame, $Q=q_{1}+q_{2}$, and $q=q_{1}-q_{2}$.

The phase-space measure $d \Phi_{2}$ can be parametrized as follows:

$$
\begin{aligned}
d \Phi_{2}= & \frac{1}{64(2 \pi)^{5}} d Q^{2} \theta\left(m_{\tau}^{2}-Q^{2}\right) \theta\left(Q^{2}-4 m_{\pi}^{2}\right) d \Omega_{Q} \\
& \times \frac{\lambda^{1 / 2}\left(m_{\tau}^{2}, Q^{2}, 0\right)}{m_{\tau}^{2}} d \Omega_{1}^{*} \frac{\lambda^{1 / 2}\left(Q^{2}, m_{\pi}^{2} m_{\pi}^{2}\right)}{Q^{2}},
\end{aligned}
$$

where $d \Omega_{Q}=d \cos \theta_{Q} d \varphi_{Q}$ is the solid angle element of $Q$, i.e., of $\rho\left(\rho^{\prime}\right)$, in the $\tau$ rest frame and $d \Omega_{1}^{*}$ is the solid angle element of the charged pion $\pi^{-}\left(q_{1}\right)$ in the rest frame of $\rho\left(\rho^{\prime}\right)$. Moreover,

$$
\lambda(x, y, z)=x^{2}+y^{2}+z^{2}-2 x y-2 x z-2 y z .
$$

The form factor $F_{\pi}$ in (A12) can be parametrized by [59]

$$
F_{\pi}\left(Q^{2}\right)=\frac{B_{\rho}\left(Q^{2}\right)+\beta_{2} B_{\rho^{\prime}}\left(Q^{2}\right)}{1+\beta_{2}},
$$

where

$$
B_{\rho}(x)=\frac{m_{\rho}^{2}}{m_{\rho}^{2}-x-i m_{\rho} \Gamma_{\rho}(x)} \quad \text { and } \rho \rightarrow \rho^{\prime} .
$$

The label $\rho\left(\rho^{\prime}\right)$ refers to the $\rho\left(\rho^{\prime}\right)$ resonance and $\beta_{2}$ is a tuning parameter (see below).

We use for the energy-dependent off-shell widths of the $\rho$ and $\rho^{\prime}$ that are needed in (A18)

$$
\Gamma_{\rho}(x)=\Gamma_{\rho}\left(m_{\rho}^{2}\right) \frac{m_{\rho}}{\sqrt{x}}\left(\frac{p(x)}{p\left(m_{\rho}^{2}\right)}\right)^{3} \theta\left(x-4 m_{\pi}^{2}\right),
$$

where 


$$
p(x)=\frac{1}{2} \sqrt{x-4 m_{\pi}^{2}}
$$

and $\Gamma_{\rho^{\prime}}(x)$ is given by the same formula with label $\rho \rightarrow \rho^{\prime}$. A value for the on-shell width $\Gamma_{\rho}\left(m_{\rho}^{2}\right)$ and $\Gamma_{\rho^{\prime}}\left(m_{\rho^{\prime}}^{2}\right)$, respectively, is given in (A20).

We use the following input values for the computations of the optimal $C P$ observables in Sec. V:

$$
\begin{aligned}
m_{\tau} & =1.777 \mathrm{GeV}, \quad m_{\pi}=0.140 \mathrm{GeV}, \\
m_{\rho} & =0.775 \mathrm{GeV}, \quad m_{\rho^{\prime}}=1.465 \mathrm{GeV}, \\
G_{F} & =1.1664 \times 10^{-5}(\mathrm{GeV})^{-2}, \quad V_{u d}=0.974, \\
\Gamma_{\rho} & =0.149 \mathrm{GeV}, \quad \Gamma_{\rho^{\prime}}=0.400 \mathrm{GeV} .
\end{aligned}
$$

With this input, agreement with the experimental width $\Gamma\left(\tau^{-} \rightarrow \pi^{-} \pi^{0} \nu_{\tau}\right)_{\exp }=5.78 \times 10^{-13} \mathrm{GeV}$ is obtained when the tuning parameter $\beta_{2}$ in Eq. (A17) is chosen to be

$$
\beta_{2}=-0.175 \text {. }
$$

The differential decay density matrix for the chargeconjugate decay

$$
\tau^{+}(k) \rightarrow \pi^{+}\left(q_{1}\right) \pi^{0}\left(q_{2}\right) \bar{\nu}_{\tau}
$$

is of the same form as (A11) with the squared matrix element

$$
\left|\mathcal{M}_{2}^{\prime}\right|^{2}=G_{F}^{2}\left|V_{u d}\right|^{2}\left|F_{\pi}\left(Q^{2}\right)\right|^{2}\left(A_{2} \rrbracket-\boldsymbol{H}_{2} \cdot \boldsymbol{\sigma}\right),
$$

and $A_{2}$ and $\boldsymbol{H}_{2}$ are given in Eqs. (A13) and (A14), respectively.

One may also determine the $\tau$-spin analyzing power of the "resonance" $Q^{\mp}$ in the $\tau^{\mp} \rightarrow \pi^{\mp} \pi^{0} \nu_{\tau}$ decay mode by computing the following decay density matrix:

$d x \frac{d \Omega_{Q}}{4 \pi} \mathcal{D}^{Q^{\mp}}=d x \frac{d \Omega_{Q}}{4 \pi}\left[a_{2, Q}(x) \mathbb{1} \pm b_{2, Q}(x) \hat{\boldsymbol{Q}} \cdot \boldsymbol{\sigma}\right]$,

where $4\left(m_{\pi} / m_{\tau}\right)^{2} \leq x \equiv Q^{2} / m_{\tau}^{2} \leq 1$ and $\hat{\boldsymbol{Q}}=\left(\boldsymbol{q}_{1}+\boldsymbol{q}_{2}\right) \mid /$ $\left|\left(\boldsymbol{q}_{1}+\boldsymbol{q}_{2}\right)\right|$. The spectral functions $a_{2, Q}$ and $b_{2, Q}$ are shown in Fig. 5. Integrating the right-hand side of (A23) over $x$ the decay density matrix takes the form (A4) with $\alpha_{a} \rightarrow \alpha_{2, Q}$ and $\hat{\mathbf{q}}_{\mp} \rightarrow \hat{\boldsymbol{Q}}$. We get for $\alpha_{2, Q}$

$$
\alpha_{2, Q}=0.42
$$

Comparison with (A10) shows that taking into account the finite widths of the intermediate resonances and the whole kinematic range of $Q^{2}$ leads to a slightly smaller $\tau$-spin analyzing power. Nevertheless, we will use the value (A10) in the computation of the expectation values of the simple $C P$ observables in Sec. V.

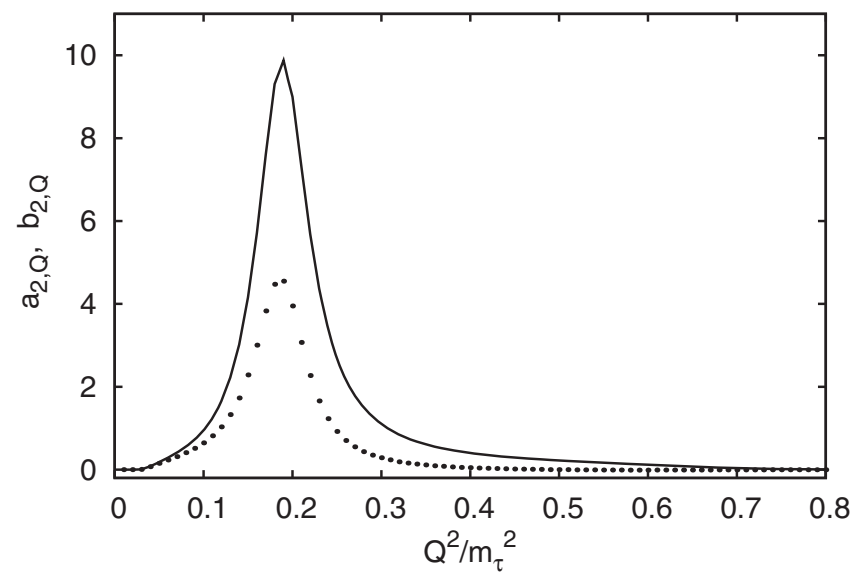

FIG. 5. The spectral functions $a_{2, Q}$ (solid curve) and $b_{2, Q}$ (dotted curve) defined in Eq. (A23).

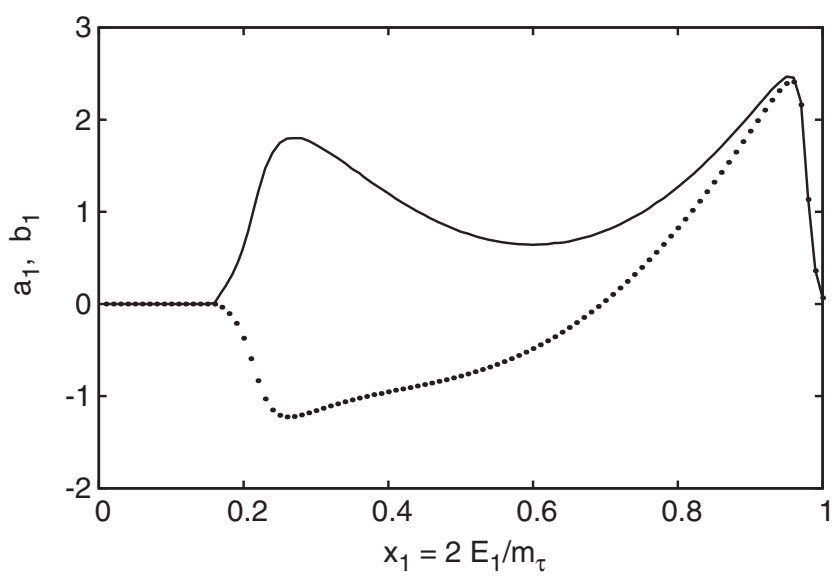

FIG. 6. The spectral functions $a_{1}$ (solid curve) and $b_{1}$ (dotted curve) defined in Eq. (A25).

For completeness we determine also the $\tau$-spin analyzing power of the charged pion in $\tau^{\mp} \rightarrow \pi^{\mp}\left(q_{1}\right)+\pi^{0} \nu_{\tau}$. The respective 1-prong decay density matrix is given by

$$
\begin{aligned}
& \frac{d^{3} q_{1}}{(2 \pi)^{3} 2 E_{1}} \mathcal{D}^{\pi^{\mp}}\left(\tau^{\mp} \rightarrow \pi^{\mp}\left(q_{1}\right)+\pi^{0} \nu_{\tau}\right) \\
& \quad=d x_{1} \frac{d \Omega_{1}}{4 \pi}\left[a_{1}\left(x_{1}\right) \mathbb{1} \pm b_{1}\left(x_{1}\right) \hat{\mathbf{q}}_{1} \cdot \boldsymbol{\sigma}\right],
\end{aligned}
$$

where $x_{1}=2 E_{1} / m_{\tau}$ and $2 m_{\pi} / m_{\tau} \leq x_{1} \leq 1$. The spectral functions $a_{1}$ and $b_{1}$ are shown in Fig. 6. Integrating the right-hand side of (A25) over $x_{1}$ the decay density matrix takes the form (A4) with $\alpha_{a} \rightarrow \alpha_{1}$ and $\hat{\mathbf{q}}_{\mp} \rightarrow \hat{\mathbf{q}}_{1}$. We get for the $\tau$-spin analyzing power $\alpha_{1}$ of the charged pion ${ }^{9}$

\footnotetext{
${ }^{9}$ This decay mode was analyzed in [9] using only the intermediate $\rho$ in the narrow-width approximation.
} 


$$
\alpha_{1}=-0.036
$$

Figure 6 shows that negative and positive contributions cancel to a large extent when $b_{1}$ is integrated over the whole kinematic range, leading to the small value (A26). The value of $\alpha_{1}$ can be enhanced by a suitable cut on $x_{1}$. We do not use (A25) in our analysis of Sec. V.

5. The decay $\tau^{\mp} \rightarrow a_{1}^{\mp} \rightarrow \pi^{\mp}\left(q_{1}\right)+\pi^{\mp}\left(q_{2}\right)+\pi^{ \pm}\left(q_{3}\right)+\nu_{\tau}$

The decay mode to three charged prongs proceeds mainly via an intermediate $a_{1}$ resonance. If one approximates the $\tau \rightarrow 3 \pi$ decay mode by $\tau$ decay to an on-shell $a_{1}$, the $\tau$-spin analyzing power of this resonance would be maximal, as stated above, if the $a_{1}$ polarization states can be separated efficiently [34,58]. If one sums over the $a_{1}$ polarizations, the $\tau \rightarrow a_{1} \nu_{\tau}$ decay density matrix is of the form (A3) and (A9) with the label $\rho \rightarrow a_{1}$. The $a_{1}$ mass is not precisely determined but, in any case, the $\tau$-spin analyzing power of this resonance is poor in the on-shell approximation. Using (A9) (with $m_{\rho} \rightarrow m_{a_{1}}$ ) with the value $m_{a_{1}}=1.230 \mathrm{GeV}$ given by the Particle Data Group [1] one obtains $\alpha_{a_{1}}=0.02$.

However, maximal sensitivity to the $\tau$ polarization can be obtained with the $3 \pi$ decay mode if the full decay dynamics is exploited and the energies and momenta of the three pions are measured. We use the $\tau \rightarrow 3 \pi \nu_{\tau}$ matrix element given in [59] (cf. also [60,61]) where this decay is described by the decay chain $\tau \rightarrow a_{1} \rightarrow \rho\left(\rho^{\prime}\right) \pi \rightarrow 3 \pi$ with off-shell intermediate resonances. Exact isospin invariance is assumed. ${ }^{10}$ We obtain for the differential $\tau^{-} \rightarrow 2 \pi^{-} \pi^{+} \nu_{\tau}$ decay density matrix $\mathcal{D}^{3 \pi}$ in the $\tau^{-}$rest frame with the normalization conventions (31) and (32)

$$
\prod_{i=1}^{3} \frac{d^{3} q_{i}}{(2 \pi)^{3} 2 q_{i}^{0}} \mathcal{D}^{3 \pi}\left(\tau^{-}(k) \rightarrow \pi^{-}\left(q_{1}\right) \pi^{-}\left(q_{2}\right) \pi^{+}\left(q_{3}\right) \nu_{\tau}\right)=\frac{1}{2 m_{\tau} \Gamma_{3 \pi}} d \Phi_{3}\left|\mathcal{M}_{3}\right|^{2},
$$

where $\Gamma_{3 \pi}=\Gamma\left(\tau^{-} \rightarrow \pi^{-} \pi^{-} \pi^{+} \nu_{\tau}\right)$ and the phase-space measure is given in the recursive phase-space parametrization by

$$
\begin{aligned}
d \Phi_{3}= & \frac{1}{2^{9}(2 \pi)^{8}} d Q^{2} d u \theta\left(m_{\tau}^{2}-Q^{2}\right) \theta\left(Q^{2}-9 m_{\pi}^{2}\right) \theta\left(\left(\sqrt{Q^{2}}-m_{\pi}\right)^{2}-u\right) \theta\left(u-4 m_{\pi}^{2}\right) \\
& \times d \Omega_{Q} \frac{\lambda^{1 / 2}\left(m_{\tau}^{2}, Q^{2}, 0\right)}{m_{\tau}^{2}} d \Omega_{3}^{*} \frac{\lambda^{1 / 2}\left(Q^{2}, u, m_{\pi}^{2}\right)}{Q^{2}} d \Omega_{2}^{* *} \frac{\lambda^{1 / 2}\left(u, m_{\pi}^{2}, m_{\pi}^{2}\right)}{u} .
\end{aligned}
$$

Here $Q=q_{1}+q_{2}+q_{3}, u=\left(q_{1}+q_{2}\right)^{2}$, and $d \Omega_{Q}=d \cos \theta_{Q} d \varphi_{Q}$ is the solid angle element of $Q$, i.e., $a_{1}$, in the $\tau$ rest frame, $d \Omega_{3}^{*}$ is the solid angle element of $\pi^{+}\left(q_{3}\right)$ in the rest frame of $a_{1}$, and $d \Omega_{2}^{* *}$ is the solid angle element of $\pi^{0}\left(q_{2}\right)$ in the rest frame of $\rho$, i.e., the zero-momentum frame of $q_{1}+q_{2}$. Note that the statistics factor $1 / 2$ for two identical particles in the final state is compensated here by the normalization convention (32). The squared matrix element is given by

$$
\left|\mathcal{M}_{3}\right|^{2}=G_{F}^{2}\left|V_{u d}\right|^{2}\left(A_{3} \mathbb{1}+\boldsymbol{H}_{3} \cdot \boldsymbol{\sigma}\right)
$$

where

$$
\begin{aligned}
& A_{3}=\left|F_{1}\right|^{2}\left[4\left(k \cdot V_{1}\right)^{2}-2\left(k \cdot Q-Q^{2}\right) V_{1}^{2}\right]+\left|F_{2}\right|^{2}\left[4\left(k \cdot V_{2}\right)^{2}-2\left(k \cdot Q-Q^{2}\right) V_{2}^{2}\right] \\
&+\operatorname{Re}\left(F_{1} F_{2}^{*}\right)\left[8 k \cdot V_{1} k \cdot V_{2}-4\left(k \cdot Q-Q^{2}\right) V_{1} \cdot V_{2}\right]-2 i\left(F_{2} F_{1}^{*}-F_{1} F_{2}^{*}\right) \epsilon\left(k, Q, V_{1}, V_{2}\right), \\
& H_{3}^{j}= 2 m_{\tau}\left\{\left|F_{1}\right|^{2}\left[2 k \cdot V_{1} V_{1}^{j}+V_{1}^{2} Q^{j}\right]+\left|F_{2}\right|^{2}\left[2 k \cdot V_{2} V_{2}^{j}+V_{2}^{2} Q^{j}\right]\right. \\
&\left.+2 \operatorname{Re}\left(F_{1} F_{2}^{*}\right)\left[k \cdot V_{2} V_{1}^{j}+k \cdot V_{1} V_{2}^{j}+V_{1} \cdot V_{2} Q^{j}\right]\right\} \\
&+2 i m_{\tau}\left(F_{2} F_{1}^{*}-F_{1} F_{2}^{*}\right) \epsilon\left(q^{\prime}, j, V_{1}, V_{2}\right),
\end{aligned}
$$

and $j=1,2,3, k=\left(m_{\tau}, \mathbf{0}\right)^{T}, q^{\prime}=k-Q$,

$$
V_{1}^{\mu}=\left(g^{\mu \nu}-\frac{Q^{\mu} Q^{\nu}}{Q^{2}}\right)\left(q_{1}-q_{3}\right)_{\nu}, \quad V_{2}^{\mu}=\left(g^{\mu \nu}-\frac{Q^{\mu} Q^{\nu}}{Q^{2}}\right)\left(q_{2}-q_{3}\right)_{\nu},
$$

\footnotetext{
${ }^{10}$ The $\tau \rightarrow 3 \pi \nu_{\tau}$ decay was analyzed in [62] within the resonance chiral theory using an elaborate description of the $a_{1}$ off-shell width.
} 
and $\epsilon\left(k, Q, V_{1}, V_{2}\right)=\epsilon_{\mu \nu \alpha \beta} k^{\mu} Q^{\nu} V_{1}^{\alpha} V_{2}^{\beta}, \epsilon\left(q^{\prime}, j, V_{1}, V_{2}\right)=$ $\epsilon_{\mu j \alpha \beta} q^{\prime \mu} V_{1}^{\alpha} V_{2}^{\beta}$, and we use the convention $\epsilon_{0123}=+1$. Moreover,

$$
F_{1}=F\left(Q^{2}, s\right), \quad F_{2}=F\left(Q^{2}, t\right),
$$

where $s=\left(q_{1}+q_{3}\right)^{2}$ and $t=\left(q_{2}+q_{3}\right)^{2}$. The function $F$ is given by [59]

$$
F\left(Q^{2}, x\right)=\frac{2 \sqrt{2}}{3 f_{\pi}} B_{a_{1}}\left(Q^{2}\right) F_{\pi}(x),
$$

where $f_{\pi}$ is the pion decay constant (in the convention $f_{\pi}=0.093 \mathrm{GeV}$ ) and $B_{a_{1}}$ denotes the Breit-Wigner enhancement factor of the $a_{1}$ meson:

$$
B_{a_{1}}\left(Q^{2}\right)=\frac{m_{a_{1}}^{2}}{m_{a_{1}}^{2}-Q^{2}-i m_{a_{1}} \Gamma_{a_{1}}\left(Q^{2}\right)} .
$$

We use as a model for the energy-dependent off-shell width of the $a_{1}$ meson

$$
\Gamma_{a_{1}}\left(Q^{2}\right)=\Gamma_{a_{1}}\left(m_{a_{1}}^{2}\right) \frac{g\left(Q^{2}\right)}{g\left(m_{a_{1}}^{2}\right)},
$$

where $\Gamma_{a_{1}}\left(m_{a_{1}}^{2}\right)$ is the on-shell width (see below) and the function $g$ is given in Eq. (3.16) of Ref. [59]. Moreover, the pion "form factor" $F_{\pi}(x)$ is given by the formulas (A17)(A19) above where now the tuning parameter $\beta_{2}$ is to be replaced by $\beta_{3}$ that will be determined below.

The differential decay density matrix for the chargeconjugate decay

$$
\tau^{+}(k) \rightarrow \pi^{+}\left(q_{1}\right) \pi^{+}\left(q_{2}\right) \pi^{-}\left(q_{3}\right) \bar{\nu}_{\tau}
$$

is of the same form as Eqs. (A27) with the squared matrix element

$$
\left|\mathcal{M}_{3}^{\prime}\right|^{2}=G_{F}^{2}\left|V_{u d}\right|^{2}\left(A_{3} \mathbb{1}-\boldsymbol{H}_{3} \cdot \boldsymbol{\sigma}\right),
$$

and $A_{3}$ and $\boldsymbol{H}_{3}$ are given in Eqs. (A30) and (A31), respectively.

To the best of our knowledge the differential $\tau \rightarrow 3 \pi \nu$ density matrix (A27)-(A31) was so far not given in this explicit form in the literature.

For our computation of the expectation values of the optimal observables in Sec. V we use the above formulas with the input values (A20) and

$f_{\pi}=0.093 \mathrm{GeV}, \quad m_{a_{1}}=1.230 \mathrm{GeV}, \quad \Gamma_{a_{1}}=0.483 \mathrm{GeV}$.

It remains to fix the tuning parameter $\beta_{3}$. Using the above squared matrix element and input parameters we find agreement with the experimental width $\Gamma\left(\tau^{-} \rightarrow\right.$ $\left.2 \pi^{-} \pi^{+} \nu_{\tau}\right)_{\exp }=2.11 \times 10^{-13} \mathrm{GeV}$ when the tuning parameter $\beta_{3}$ is chosen to be

$$
\beta_{3}=-0.204
$$

\section{The decay $\tau^{\mp} \rightarrow a_{1}^{\mp} \rightarrow \pi^{0}\left(q_{1}\right)+\pi^{0}\left(q_{2}\right)+\pi^{\mp}\left(q_{3}\right)+\nu_{\tau}$}

For completeness we discuss here also this decay mode, although we do not use it in the analysis of Sec. V. Assuming exact isospin invariance the differential decay density matrices for $\tau^{\mp} \rightarrow 2 \pi^{\mp} \pi^{ \pm} \nu_{\tau}$ derived in the previous subsection can be used also for these decay modes. Using the above input parameters with the exception $m_{\pi}=m_{\pi^{+}}=$ $0.140 \mathrm{GeV} \rightarrow m_{\pi}=m_{\pi^{0}}=0.135 \mathrm{GeV}$, agreement with the experimental width $\Gamma\left(\tau^{-} \rightarrow 2 \pi^{0} \pi^{-} \nu_{\tau}\right)_{\exp }=2.10 \times$ $10^{-13} \mathrm{GeV}$ is obtained with the following value of the tuning parameter, here denoted by $\beta_{3}^{\prime}$ :

$$
\beta_{3}^{\prime}=-0.190
$$

Moreover, the $\tau$-spin analyzing power of the charged pion in this decay mode is also of interest. The 1-prong decay density matrix for $\tau^{\mp} \rightarrow \pi^{\mp}\left(q_{3}\right)+2 \pi^{0} \nu_{\tau}$, normalized to the charged particle multiplicity $n_{\pi^{ \pm}}=1$, is given by

$$
\begin{aligned}
& \frac{d^{3} q_{3}}{(2 \pi)^{3} 2 E_{3}} \mathcal{D}^{\pi^{\mp}}\left(\tau^{\mp} \rightarrow \pi^{\mp}\left(q_{3}\right)+2 \pi^{0} \nu_{\tau}\right) \\
& =d x_{3} \frac{d \Omega_{3}}{4 \pi}\left[a_{3}\left(x_{3}\right) \mathbb{1} \pm b_{3}\left(x_{3}\right) \hat{\mathbf{q}}_{3} \cdot \boldsymbol{\sigma}\right],
\end{aligned}
$$

where $x_{3}=2 E_{3} / m_{\tau}$ and $2 m_{\pi} / m_{\tau} \leq x_{3} \leq 1-3\left(m_{\pi} / m_{\tau}\right)^{2}$. The spectral functions $a_{3}$ and $b_{3}$ are shown in Fig. 7 . Integrating the right-hand side of (A41) over $x_{3}$ the decay density matrix takes the form (A4) with $\alpha_{a} \rightarrow \alpha_{3}$ and

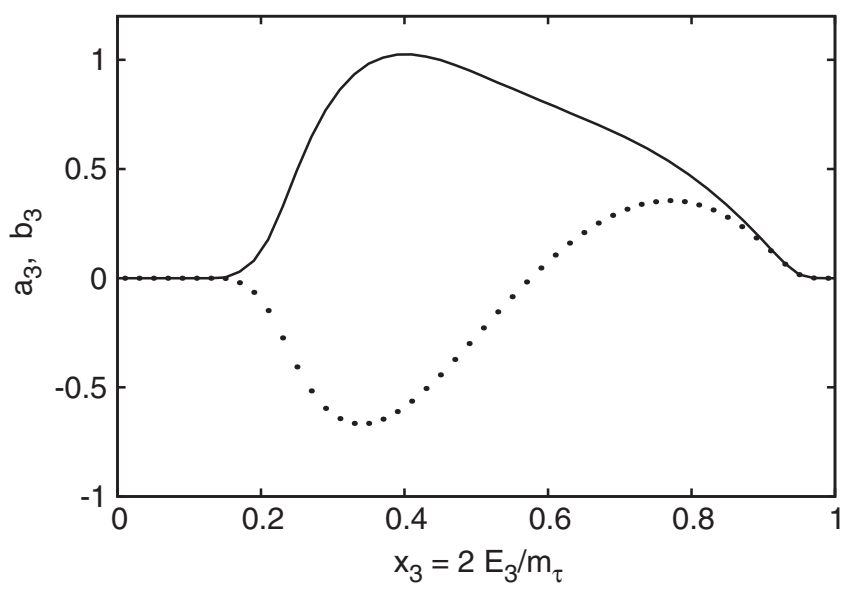

FIG. 7. The spectral functions $a_{3}$ (solid curve) and $b_{3}$ (dotted curve) defined in Eq. (A41). 
$\hat{\mathbf{q}}_{\mp} \rightarrow \hat{\mathbf{q}}_{3}$. We get for the $\tau$-spin analyzing power $\alpha_{3}$ of the charged pion ${ }^{11}$

$$
\alpha_{3}=-0.144
$$

This number is rather small because $b_{3}$ has both negative and positive contributions that cancel to a large extent when integrated over $x_{3}$. The analyzing power can be enhanced by a suitable cut on $x_{3}$.

\section{APPENDIX B: EXPECTATION VALUES AND COVARIANCES OF CP-ODD OBSERVABLES}

In this Appendix we discuss general properties of expectation values and covariances of the $C P$-odd observables introduced in Sec. IV and computed in Sec. V. We treat first case (i) of Sec. III where only one charged particle is measured from $\tau^{-}$and $\tau^{+}$decays, respectively. The differential cross section of the two-particle inclusive reaction (28) and (29) as used in this paper is given by (30):

$$
\begin{aligned}
d \sigma_{a \bar{b}}= & \frac{\sqrt{1-4 m_{\tau}^{2} / s}}{16 \pi s} \operatorname{Br}\left(\tau^{-} \rightarrow A\right) \operatorname{Br}\left(\tau^{+} \rightarrow \bar{B}\right) \\
& \times \operatorname{Tr}\left[R \mathcal{D}^{a} \mathcal{D}^{\bar{b}}\right] \frac{\left|\mathbf{q}_{-}^{*}\right|}{(2 \pi)^{2}} \frac{\left|\mathbf{q}_{+}^{*}\right|}{(2 \pi)^{2}} d E_{-}^{*} d E_{+}^{*} \frac{d \Omega_{k_{+}}}{4 \pi} \frac{d \Omega_{-}^{*}}{4 \pi} \frac{d \Omega_{+}^{*}}{4 \pi},
\end{aligned}
$$

where we have used in (30) the momenta of the charged particles $a$ and $\bar{b}$ and the corresponding phase-space measures in the respective $\tau^{-}$and $\tau^{+}$rest frame. The one-particle inclusive decay density matrices in the $\tau^{\mp}$ rest frames are given in (A3). We recall the relation between the respective rest-frame momenta $q_{\mp}^{*}$ and $k_{\mp}^{*}=\left(m_{\tau}, \mathbf{0}\right)^{T}$ and the momenta $q_{\mp}$ and $k_{\mp}$ in the $e^{+} e^{-}$c.m. frame. With the Lorentz boost

$$
\Lambda_{\mathbf{k}}=\left(\begin{array}{cc}
\frac{k^{0}}{m_{\tau}} & \frac{k^{j}}{m_{\tau}} \\
\frac{k^{i}}{m_{\tau}} & \delta^{i j}+\hat{k}^{i} \hat{k}^{j}\left(\frac{k^{0}-m_{\tau}}{m_{\tau}}\right)
\end{array}\right),
$$

where $\mathbf{k}$ is the three-momentum of $\tau^{+}$in the $e^{+} e^{-}$c.m. frame, we have

$$
\Lambda_{ \pm \mathbf{k}} k_{\mp}=k_{\mp}^{*}, \quad \Lambda_{ \pm \mathbf{k}} q_{\mp}=q_{\mp}^{*} .
$$

Next we decompose (B1) according to (51) and (52), neglecting terms quadratic in $\hat{d}_{\tau}$. Here our phase-space variables are

$$
\phi=\left(E_{-}^{*}, E_{+}^{*}, \hat{\mathbf{k}}, \hat{\mathbf{q}}_{-}^{*}, \hat{\mathbf{q}}_{+}^{*}\right)
$$

\footnotetext{
${ }^{11} \mathrm{~A}$ simpler description of this decay mode was used in [9] and the value $\alpha_{\pi}=-0.18$ was obtained.
}

and the measure is

$$
d \phi=d E_{-}^{*} d E_{+}^{*} \frac{d \Omega_{k}}{4 \pi} \frac{d \Omega_{-}^{*}}{4 \pi} \frac{d \Omega_{+}^{*}}{4 \pi} .
$$

We get

$$
d \sigma_{a \bar{b}}=\left\{S_{\mathrm{SM}}^{a \bar{b}}(\phi)+S_{C P, R}^{a \bar{b}}(\phi) \operatorname{Re} \hat{d}_{\tau}+S_{C P, I}^{a \bar{b}}(\phi) \operatorname{Im} \hat{d}_{\tau}\right\} d \phi,
$$

where, using (A3),

$$
\begin{aligned}
S_{\mathrm{SM}}^{a \bar{b}}(\phi)= & \frac{\sqrt{1-4 m_{\tau}^{2} / s}}{16 \pi s} \operatorname{Br}\left(\tau^{-} \rightarrow A\right) \\
& \times \operatorname{Br}\left(\tau^{+} \rightarrow \bar{B}\right) \frac{\chi_{\mathrm{SM}, \alpha \alpha^{\prime} \beta \beta^{\prime}}}{\left|1+e^{2} \Pi_{c}(s)\right|^{2}} \\
& \times n_{a}\left(E_{-}^{*}\right)\left[\delta_{\beta^{\prime} \beta}+h_{a}\left(E_{-}^{*}\right) \hat{\mathbf{q}}_{-}^{*} \cdot \boldsymbol{\sigma}_{\beta^{\prime} \beta}\right] \\
& \times n_{b}\left(E_{+}^{*}\right)\left[\delta_{\alpha^{\prime} \alpha}-h_{b}\left(E_{+}^{*}\right) \hat{\mathbf{q}}_{+}^{*} \cdot \boldsymbol{\sigma}_{\alpha^{\prime} \alpha}\right]
\end{aligned}
$$

The quantities $S_{C P, R}^{a \bar{b}}$ and $S_{C P, I}^{a \bar{b}}$ are obtained from (B7) by the replacements

$$
\chi_{\mathrm{SM}} \rightarrow \chi_{C P}^{R} \quad \text { and } \quad \chi_{\mathrm{SM}} \rightarrow \chi_{C P}^{I},
$$

respectively; see (38)-(40).

We can now perform the traces in (B7). With (43) we see that this amounts to make the following replacements in (38)-(40):

$$
\begin{aligned}
\mathbb{1} & \rightarrow 4 n_{b} n_{a}, \\
\boldsymbol{\sigma}_{+} & \rightarrow-4 n_{b} h_{b} n_{a} \hat{\mathbf{q}}_{+}^{*}, \\
\boldsymbol{\sigma}_{-} & \rightarrow 4 n_{b} n_{a} h_{a} \hat{\mathbf{q}}_{-}^{*}, \\
\sigma_{+}^{r} \sigma_{-}^{s} & \rightarrow-4 n_{b} h_{b} n_{a} h_{a} \hat{q}_{+}^{* r} \hat{q}_{-}^{* s} .
\end{aligned}
$$

Next, we consider the transformation

$$
\mathbf{k} \rightarrow-\mathbf{k}, \quad \mathbf{q}_{+} \rightarrow-\mathbf{q}_{+}, \quad \mathbf{q}_{-} \rightarrow-\mathbf{q}_{-},
$$

which, using (B3), implies

$$
\hat{\mathbf{k}} \rightarrow-\hat{\mathbf{k}}, \quad \hat{\mathbf{q}}_{+}^{*} \rightarrow-\hat{\mathbf{q}}_{+}^{*}, \quad \hat{\mathbf{q}}_{-}^{*} \rightarrow-\hat{\mathbf{q}}_{-}^{*},
$$

and vice versa. These transformations correspond to the naive "time reversal" transformation ${ }^{12} T_{N}$ referred to in Sec. III. Inspection of $\chi_{\mathrm{SM}}, \chi_{C P}^{R}$, and $\chi_{C P}^{I}$, i.e., of Eqs. (38)(40) with the replacements (B9), shows that applying (B11) we have

\footnotetext{
${ }^{12}$ One may also transform $\mathbf{p} \rightarrow-\mathbf{p}$, but this is irrelevant here.
} 


$$
\begin{aligned}
S_{\mathrm{SM}}^{a \bar{b}}(\phi) & \rightarrow S_{\mathrm{SM}}^{a \bar{b}}(\phi), \quad S_{C P, R}^{a \bar{b}}(\phi) \rightarrow-S_{C P, R}^{a \bar{b}}(\phi), \\
S_{C P, I}^{a \bar{b}}(\phi) & \rightarrow S_{C P, I}^{a \bar{b}}(\phi) .
\end{aligned}
$$

We turn to the simple and optimal observables of Sec. IV. We assume integration over the whole phase space or, if cuts are applied, we assume the cuts to be $C P$-symmetric. In addition, we assume the cuts to be invariant under (B10). The tensors $\hat{T}^{i j}$ and $T^{i j}$ of (44) and (45) are odd, whereas $\hat{Q}^{i j}$ and $Q^{i j}$ of (46) and (47) are even under the transformation (B10).

Let us first consider the case $a=b$. The $C P$ properties of the observables $T$ and $Q$ imply

$$
\begin{array}{ll}
E_{0}\left(T^{i j}\right)=0, & E_{0}\left(\hat{T}^{i j}\right)=0, \\
E_{0}\left(Q^{i j}\right)=0, & E_{0}\left(\hat{Q}^{i j}\right)=0 .
\end{array}
$$

Moreover, turning to the covariance matrix of one of the $T$ and one of the $Q$ variables, the transformation (B10) implies

$$
E_{0}\left(T^{i j} Q^{k l}\right)=0
$$

and likewise for the other $T Q$ correlations. That is, the covariance matrix of the $T, Q$ variables is diagonal.

The optimal $C P$ observables are in the case $a=b$

$$
\mathcal{O}_{R}^{a \bar{a}}(\phi)=\frac{S_{C P, R}^{a \bar{a}}(\phi)}{S_{\mathrm{SM}}^{a \bar{a}}(\phi)}, \quad \mathcal{O}_{I}^{a \bar{a}}(\phi)=\frac{S_{C P, I}^{a \bar{a}}(\phi)}{S_{\mathrm{SM}}^{a \bar{a}}(\phi)}
$$

The $C P$ transformation properties of these observables imply

$$
E_{0}\left(\mathcal{O}_{R}^{a \bar{a}}\right)=0, \quad E_{0}\left(\mathcal{O}_{I}^{a \bar{a}}\right)=0,
$$

and applying the $T_{N}$ transformation (B10) it follows that

$$
E_{0}\left(\mathcal{O}_{R}^{a \bar{a}} \mathcal{O}_{I}^{a \bar{a}}\right)=0
$$

Thus, the covariance matrix is diagonal in this case:

$$
V\left(\mathcal{O}^{a \bar{a}}\right)=\left(\begin{array}{cc}
E_{0}\left(\mathcal{O}_{R}^{a \bar{a}} \mathcal{O}_{R}^{a \bar{a}}\right) & 0 \\
0 & E_{0}\left(\mathcal{O}_{I}^{a \bar{a}} \mathcal{O}_{I}^{a \bar{a}}\right)
\end{array}\right)
$$

Next we turn to the case $a \neq b$. As this final state is no longer $C P$-symmetric, the $C P$ transformation properties of the observables are no longer of immediate use. Let us first consider the simple observables, for instance, $T^{i j}$ and $\hat{Q}^{i j}$. Applying the $T_{N}$ transformation (B10) we get

$$
E_{0}^{a \bar{b}}\left(T^{i j}\right) \equiv\left\langle T^{i j}\right\rangle_{0, a \bar{b}}=0
$$

The transformation (B10) implies also that expectation values of the form (B14) vanish in the nondiagonal case. As to the SM expectation value

$$
E_{0}^{a \bar{b}}\left(\hat{Q}^{i j}\right) \equiv\left\langle\hat{Q}^{i j}\right\rangle_{0, a \bar{b}}
$$

there is, however, in the case $a \neq b$ no symmetry argument implying that it vanishes, too. Therefore, one should use in this case in general the observables

$$
\hat{Q}^{\prime i j}=\hat{Q}^{i j}-\left\langle\hat{Q}^{i j}\right\rangle_{0, a \bar{b}}
$$

From the $C P$ property of $\hat{Q}^{i j}$ one gets, of course,

$$
\left\langle\hat{Q}^{i j}\right\rangle_{0, a \bar{b}}+\left\langle\hat{Q}^{i j}\right\rangle_{0, b \bar{a}}=0
$$

Thus, the respective quantity to probe for $C P$ violation is (49),

$$
\frac{1}{2}\left\{\left\langle\hat{Q}^{i j}\right\rangle_{a \bar{b}}+\left\langle\hat{Q}^{i j}\right\rangle_{b \bar{a}}\right\}
$$

But the corresponding variance in the SM, for instance of the $i=j=3$ components, has to be calculated in general as

$$
\left\langle\hat{Q}^{\prime 33} \hat{Q}^{\prime 33}\right\rangle_{0, a \bar{b}}=\left\langle\hat{Q}^{\prime 33} \hat{Q}^{\prime 33}\right\rangle_{0, b \bar{a}}
$$

The above statements apply, of course, also to $Q^{i j}$. Yet in our analysis where we use the SM matrix element of the form (B7) and integrate over the whole phase space we find that (B20) vanishes within our numerical uncertainties of order $10^{-4}$. This holds also for the respective expectation values of $Q^{i j}$.

The optimal observables are in the case $a \neq b$ [cf. (86)]:

$$
\mathcal{O}_{R}^{a \bar{b}}(\phi)=\frac{S_{C P, R}^{a \bar{b}}(\phi)}{S_{\mathrm{SM}}^{a \bar{b}}(\phi)}, \quad \mathcal{O}_{I}^{a \bar{b}}(\phi)=\frac{S_{C P, I}^{a \bar{b}}(\phi)}{S_{\mathrm{SM}}^{a \bar{b}}(\phi)},
$$

where $\mathcal{O}_{R}^{a \bar{b}}(\phi)$ and $\mathcal{O}_{I}^{a \bar{b}}(\phi)$ are odd and even under the transformation (B11), respectively; see (B12). Therefore, we have

$$
E_{0}\left(\mathcal{O}_{R}^{a \bar{b}}\right)=0
$$

For analyzing $E_{0}\left(\mathcal{O}_{I}^{a \bar{b}}\right)$ we perform in $d \sigma_{a \bar{b}}$, Eq. (B6), the variable transformation

$$
\mathbf{k} \rightarrow-\mathbf{k}
$$

The term $S_{\mathrm{SM}}^{a \bar{b}}(\phi)$ remains invariant, while $\mathcal{O}_{i}^{a \bar{b}}(\phi)(i=R, I)$ change sign; see (38)-(40) and (B7), (B8). Thus

$$
E_{0}\left(\mathcal{O}_{I}^{a \bar{b}}\right)=0
$$

if one integrates over the whole angular range of $\mathbf{k}$ or over a range that is symmetric with respect to (B27). Beyond the one-photon approximation $E_{0}\left(\mathcal{O}_{i}^{a \bar{b}}\right)(i=R, I)$ will in 
general be nonzero. Therefore, one should use in general [cf. (56)]

$$
\begin{aligned}
& \mathcal{O}_{R}^{\prime a \bar{b}}(\phi)=\mathcal{O}_{R}^{a \bar{b}}(\phi)-E_{0}\left(\mathcal{O}_{R}^{a \bar{b}}\right), \\
& \mathcal{O}_{I}^{\prime a \bar{b}}(\phi)=\mathcal{O}_{I}^{a \bar{b}}(\phi)-E_{0}\left(\mathcal{O}_{I}^{a \bar{b}}\right) .
\end{aligned}
$$

In our case here the transformations (B11) and (B27) imply that the covariance matrix $V\left(\mathcal{O}^{\prime a \bar{b}}\right)$ is still diagonal and is given by

$$
V\left(\mathcal{O}^{\prime a \bar{b}}\right)=V\left(\mathcal{O}^{a \bar{b}}\right)=\left(\begin{array}{cc}
E_{0}\left(\mathcal{O}_{R}^{a \bar{b}} \mathcal{O}_{R}^{a \bar{b}}\right) & 0 \\
0 & E_{0}\left(\mathcal{O}_{I}^{a \bar{b}} \mathcal{O}_{I}^{a \bar{b}}\right)
\end{array}\right) .
$$

This covariance matrix is then used in (75) and (77) for the estimators $\gamma_{i}$ and their covariance matrix $V(\gamma)$.

We come now to the final states of case (ii) of Sec. III to which we apply the optimal $C P$ observables. The channels where $\tau \rightarrow 3 \pi \nu_{\tau}$ is involved require a more detailed discussion. In our models for the hadronic $\tau$ decays outlined in Appendix A the squared matrix element of this decay mode given in (A29)-(A31) differs from the respective squared matrix element of $\tau \rightarrow 2 \pi \nu_{\tau}$ and those of the one-particle inclusive decays in that it contains contributions from absorptive parts caused by the finite widths of the intermediate resonances. This implies that a $T_{N}$ transformation can no longer be used to discriminate between the optimal observables $\mathcal{O}_{R}$ and $\mathcal{O}_{I}$. In order to see this explicitly let us for definiteness consider the case where $\tau^{-}$decays to three observed pions (labeled by the symbol $A$ ), while in the decay of $\tau^{+}$only one charged particle is measured (label $\bar{b}$ ). The differential cross section is obtained by inserting the respective decay density matrices into (30), taking into account (31)-(33). Using (A29) the matrix element $S_{\mathrm{SM}}^{A \bar{b}}$ is obtained from (B7), up to an overall factor, by replacing

$$
n_{a}\left(E_{-}^{*}\right)\left[\delta_{\beta^{\prime} \beta}+h_{a}\left(E_{-}^{*}\right) \hat{\mathbf{q}}_{-}^{*} \cdot \boldsymbol{\sigma}_{\beta^{\prime} \beta}\right] \rightarrow\left[A_{3} \delta_{\beta^{\prime} \beta}+\boldsymbol{H}_{3} \cdot \boldsymbol{\sigma}_{\beta^{\prime} \beta}\right] .
$$

The matrix elements $S_{C P, R}^{A \bar{b}}$ and $S_{C P, I}^{A \bar{b}}$ are obtained in the same fashion. Inspection of the functions $A_{3}$ and $H_{3}^{j}$ shows that neither has a definite behavior under the following transformation that is analogous to (B11):

$$
\mathbf{k} \rightarrow-\mathbf{k}, \quad \mathbf{q}_{i}^{*} \rightarrow-\mathbf{q}_{i}^{*} \quad(i=1,2,3) .
$$

The dispersive terms in $A_{3}$ and $H_{3}^{j}$ are even under (B32), whereas the absorptive terms are odd. Therefore, the matrix elements $S_{\mathrm{SM}}^{A \bar{b}}, S_{C P, R}^{A \bar{b}}$, and $S_{C P, I}^{A \bar{b}}$ do not have a definite transformation behavior under (B32), too. Hence we expect that

$$
E_{0}\left(\mathcal{O}_{C P, R}^{A \bar{b}} \mathcal{O}_{C P, I}^{A \bar{b}}\right) \neq 0
$$

Thus, the covariance matrix $V\left(\mathcal{O}^{\prime A} \bar{b}\right)$ can have nondiagonal elements that are nonvanishing. On the other hand, applying the transformation (B27), $\mathbf{k} \rightarrow-\mathbf{k}$, to $S_{\mathrm{SM}}^{A \bar{b}}$ and to $S_{C P, i}^{A \bar{b}}$ $(i=R, I)$ shows that the first term remains invariant while the two others change sign. Therefore, with our matrix elements we have

$$
E_{0}\left(\mathcal{O}_{C P, R}^{A \bar{b}}\right)=0, \quad E_{0}\left(\mathcal{O}_{C P, I}^{A \bar{b}}\right)=0
$$

Beyond the one-photon approximation (B34) will no longer hold. Thus when $\tau$-pair decays to final states $A \bar{b}+b \bar{A}, A \bar{A}$, and $A \bar{B}+B \bar{A}$ are considered, one should in general apply — especially in experimental analyses - the full formalism of the optimal observable method as explained in Sec. IV. The nondiagonal elements of the respective covariance matrix of the optimal $C P$ observables computed with the matrix elements for $\tau$-pair production and decay used in this paper at $\sqrt{s}=10.58 \mathrm{GeV}$ are very small and can be neglected in view of our numerical uncertainties; see Sec. V.

At last a remark that applies if the full formalism of Sec. IV has to be used. Suppose the parameters $\operatorname{Re} d_{\tau}$ and $\operatorname{Im} d_{\tau}$ have been measured in $k$ decay channels. Let us denote the results for their mean values in the channel $\kappa$ by

$$
\overline{\boldsymbol{X}}^{(\kappa)}=\left(\begin{array}{c}
\operatorname{Re} \bar{d}_{\tau}^{(\kappa)} \\
\operatorname{Im} \bar{d}_{\tau}^{(\kappa)}
\end{array}\right), \quad(\kappa=1, \ldots, k),
$$

and for the respective covariance matrix by $V^{(\kappa)}$. Furthermore, we assume these $k$ measurements to be independent, i.e., uncorrelated. We define the matrix

$$
V^{-1}=\sum_{\kappa=1}^{k}\left(V^{(\kappa)}\right)^{-1}
$$

and the overall mean

$$
\overline{\boldsymbol{X}}=V \sum_{\kappa=1}^{k}\left(V^{(\kappa)}\right)^{-1} \overline{\boldsymbol{X}}^{(\kappa)} .
$$

The covariance matrix is then given by $V$. That is, the 1 s.d. error ellipse for the mean values (B37) in the $\operatorname{Re} d_{\tau}-\operatorname{Im} d_{\tau}$ plane is given by

$$
(\boldsymbol{X}-\overline{\boldsymbol{X}})^{T} V^{-1}(\boldsymbol{X}-\overline{\boldsymbol{X}})=1 .
$$


[1] P. A. Zyla et al. (Particle Data Group), Review of particle physics, Prog. Theor. Exp. Phys. 2020, 083C01 (2020).

[2] V. Andreev et al. (ACME Collaboration), Improved limit on the electric dipole moment of the electron, Nature (London) 562, 355 (2018).

[3] G.W. Bennett et al. (Muon (g-2) Collaboration), An improved limit on the muon electric dipole moment, Phys. Rev. D 80, 052008 (2009).

[4] K. Inami et al. (Belle Collaboration), Search for the electric dipole moment of the tau lepton, Phys. Lett. B 551, 16 (2003).

[5] W. Bernreuther, U. Löw, J. P. Ma, and O. Nachtmann, $C P$ violation and Z boson decays, Z. Phys. C 43, 117 (1989).

[6] W. Bernreuther and O. Nachtmann, $C P$ Violating Correlations in Electron Positron Annihilation into $\tau$ Leptons, Phys. Rev. Lett. 63, 2787 (1989); Erratum, Phys. Rev. Lett. 64, 1072 (1990).

[7] J. G. Körner, J. P. Ma, R. Münch, O. Nachtmann, and R. Schöpf, $C P$ violating correlations in the reaction $e^{+} e^{-} \rightarrow$ hadrons, Z. Phys. C 49, 447 (1991).

[8] W. Bernreuther, G. W. Botz, O. Nachtmann, and P. Overmann, $C P$ violating effects in $\mathrm{Z}$ decays to tau leptons, $\mathrm{Z}$. Phys. C 52, 567 (1991).

[9] W. Bernreuther, O. Nachtmann, and P. Overmann, The $C P$ violating electric and weak dipole moments of the tau lepton from threshold to $500 \mathrm{GeV}$, Phys. Rev. D 48, 78 (1993).

[10] H. Albrecht et al. (ARGUS Collaboration), A search for the electric dipole moment of the tau lepton, Phys. Lett. B 485, 37 (2000).

[11] A. Stahl, Physics with tau leptons, Springer Tracts Mod. Phys. 160, 1 (2000).

[12] W. Lohmann, Electromagnetic and weak moments of the tau-lepton, Nucl. Phys. B, Proc. Suppl. 144, 122 (2005).

[13] J. Bernabeu, G. A. Gonzalez-Sprinberg, and J. Vidal, CP violation and electric-dipole-moment at low energy tau production with polarized electrons, Nucl. Phys. B763, 283 (2007).

[14] J. Bernabeu, G. A. Gonzalez-Sprinberg, J. Papavassiliou, and J. Vidal, Tau anomalous magnetic moment form-factor at super B/flavor factories, Nucl. Phys. B790, 160 (2008).

[15] S. Eidelman, D. Epifanov, M. Fael, L. Mercolli, and M. Passera, $\tau$ dipole moments via radiative leptonic $\tau$ decays, J. High Energy Phys. 03 (2016) 140.

[16] X. Chen and Y. Wu, Search for the electric dipole moment and anomalous magnetic moment of the tau lepton at tau factories, J. High Energy Phys. 10 (2019) 089.

[17] M. Dyndal, M. Klusek-Gawenda, M. Schott, and A. Szczurek, Anomalous electromagnetic moments of $\tau$ lepton in $\gamma \gamma \rightarrow \tau^{+} \tau^{-}$reaction in $\mathrm{Pb}+\mathrm{Pb}$ collisions at the $\mathrm{LHC}$, Phys. Lett. B 809, 135682 (2020).

[18] T. Abe et al. (Belle-II Collaboration), Belle II Technical Design Report, arXiv:1011.0352.

[19] E. Kou et al. (Belle-II Collaboration), The Belle II physics book, Prog. Theor. Exp. Phys. 2019, 123 C01 (2019); Erratum, Prog. Theor. Exp. Phys. 2020, 029201 (2020).

[20] M. Ablikim et al. (BESIII Collaboration), Future physics programme of BES III, Chin. Phys. C 44, 040001 (2020).

[21] D. Atwood and A. Soni, Analysis for magnetic moment and electric dipole moment form-factors of the top quark via $e^{+} e^{-} \rightarrow t \bar{t}$, Phys. Rev. D 45, 2405 (1992).
[22] M. Davier, L. Duflot, F. Le Diberder, and A. Rouge, The optimal method for the measurement of tau polarization, Phys. Lett. B 306, 411 (1993).

[23] M. Diehl and O. Nachtmann, Optimal observables for the measurement of three gauge boson couplings in $e^{+} e^{-} \rightarrow$ $W^{+} W^{-}$, Z. Phys. C 62, 397 (1994).

[24] W. Bernreuther, A. Brandenburg, and P. Overmann, CP violation beyond the standard model and tau pair production in $e^{+} e^{-}$collisions, Phys. Lett. B 391, 413 (1997); Erratum, Phys. Lett. B 412, 425 (1997).

[25] J. D. Bjorken and S. D. Drell, Relativistic Quantum Fields (Mc-Graw Hill, New York, 1965).

[26] S. Banerjee, B. Pietrzyk, J. M. Roney, and Z. Was, Tau and muon pair production cross-sections in electron-positron annihilations at $\sqrt{s}=10.58 \mathrm{GeV}$, Phys. Rev. D 77, 054012 (2008).

[27] Ia. B. Zel'dovich, Electromagnetic interaction with parity violation, Sov. Phys. JETP 6, 1184 (1958).

[28] V. V. Flambaum and I. B. Khriplovich, $P$ odd nuclear forces as a source of parity nonconservation in atoms, Sov. Phys. JETP 52, 835 (1980).

[29] V. V. Flambaum, I. B. Khriplovich, and O. P. Sushkov, On the possibility to study $P$ odd and $T$ odd nuclear forces in atomic and molecular experiments, Sov. Phys. JETP 60, 873 (1984).

[30] V. V. Flambaum, I. B. Khriplovich, and O. P. Sushkov, Nuclear anapole moments, Phys. Lett. 146B, 367 (1984).

[31] W. Buchmüller and D. Wyler, Effective Lagrangian analysis of new interactions and flavor conservation, Nucl. Phys. B268, 621 (1986).

[32] W. Bernreuther and O. Nachtmann, A note on $C P$ odd and $\mathrm{T}$ odd observables at $e^{+} e^{-}$colliders, Phys. Lett. B 268, 424 (1991).

[33] O. Nachtmann, Elementary Particle Physics: Concepts and Phenomena (Springer, Berlin, 1990).

[34] A. Rougé, Polarization observables in the 3 pi neutrino decay mode, Z. Phys. C 48, 75 (1990).

[35] J. H. Kühn, Tau polarimetry with multimeson states, Phys. Rev. D 52, 3128 (1995).

[36] J. H. Kühn, Tau kinematics from impact parameters, Phys. Lett. B 313, 458 (1993).

[37] M. Pospelov and A. Ritz, CKM benchmarks for electron electric dipole moment experiments, Phys. Rev. D 89, 056006 (2014).

[38] Y. Yamaguchi and N. Yamanaka, Large Long-Distance Contributions to the Electric Dipole Moments of Charged Leptons in the Standard Model, Phys. Rev. Lett. 125, 241802 (2020).

[39] S. M. Barr and A. Zee, Electric Dipole Moment of the Electron and of the Neutron, Phys. Rev. Lett. 65, 21 (1990); Erratum, Phys. Rev. Lett. 65, 2920 (1990).

[40] W. Bernreuther, P. Galler, C. Mellein, Z. G. Si, and P. Uwer, Production of heavy Higgs bosons and decay into top quarks at the LHC, Phys. Rev. D 93, 034032 (2016).

[41] W. Bernreuther, T. Schröder, and T. N. Pham, $C P$ violating dipole form-factors in $e^{+} e^{-} \rightarrow \bar{t} t$, Phys. Lett. B 279, 389 (1992).

[42] G. 't Hooft and M. J. G. Veltman, Scalar one loop integrals, Nucl. Phys. B153, 365 (1979). 
[43] CMS Physics Analysis summary, analysis of the $C P$ structure of the Yukawa coupling between the Higgs boson and $\tau$ leptons in proton-proton collisions at $\sqrt{s}=13 \mathrm{TeV}$, CMS-PAS-HIG-20-006.

[44] C. Caputo (CMS and ATLAS Collaborations), Searches for additional Higgs bosons decaying to tau leptons at the LHC, SciPost Phys. Proc. 1, 022 (2019).

[45] G. Aad et al. (ATLAS Collaboration), Search for Heavy Higgs Bosons Decaying Into Two Tau Leptons with the ATLAS Detector Using $p p$ Collisions at $\sqrt{s}=13 \mathrm{TeV}$, Phys. Rev. Lett. 125, 051801 (2020).

[46] M. Bauer and M. Neubert, Minimal Leptoquark Explanation for the $\mathrm{R}_{D^{(*)}}, \mathrm{R}_{K}$, and $(g-2)_{\mu}$ Anomalies, Phys. Rev. Lett. 116, 141802 (2016).

[47] E. Coluccio Leskow, G. D'Ambrosio, A. Crivellin, and D. Müller, $(g-2) \mu$, lepton flavor violation, and $Z$ decays with leptoquarks: Correlations and future prospects, Phys. Rev. D 95, 055018 (2017).

[48] A. Crivellin, M. Hoferichter, and P. Schmidt-Wellenburg, Combined explanations of $(g-2)_{\mu, e}$ and implications for a large muon EDM, Phys. Rev. D 98, 113002 (2018).

[49] I. Bigaran and R. R. Volkas, Getting chirality right: Single scalar leptoquark solution/s to the $(g-2)_{e, \mu}$ puzzle, Phys. Rev. D 102, 075037 (2020).

[50] A. Crivellin, C. Greub, D. Müller, and F. Saturnino, Scalar leptoquarks in leptonic processes, J. High Energy Phys. 02 (2021) 182.

[51] W. Dekens, J. de Vries, M. Jung, and K. K. Vos, The phenomenology of electric dipole moments in models of scalar leptoquarks, J. High Energy Phys. 01 (2019) 069.

[52] W. Bernreuther, The electric dipole moment of the muon, Z. Phys. C 56, S97 (1992).
[53] P. Poulose and S. D. Rindani, $C P$ violating dipole formfactors of the top quark and $\tau$ lepton in scalar leptoquark models, Pramana 51, 387 (1998).

[54] W. Buchmüller, R. Rückl, and D. Wyler, Leptoquarks in lepton-quark collisions, Phys. Lett. B 191, 442 (1987); Erratum, Phys. Lett. B 448, 320 (1999).

[55] A. M. Sirunyan et al. (CMS Collaboration), Search for Leptoquarks Coupled to Third-Generation Quarks in Proton-Proton Collisions at $\sqrt{s}=13 \mathrm{TeV}$, Phys. Rev. Lett. 121, 241802 (2018).

[56] M. Aaboud et al. (ATLAS Collaboration), Searches for third-generation scalar leptoquarks in $\sqrt{s}=13 \mathrm{TeV}$ pp collisions with the ATLAS detector, J. High Energy Phys. 06 (2019) 144.

[57] Y.-S. Tsai, Decay correlations of heavy leptons in $e^{+} e^{-} \rightarrow$ lepton+ lepton-, Phys. Rev. D 4, 2821 (1971); Erratum, Phys. Rev. D 13, 771 (1976).

[58] K. Hagiwara, A. D. Martin, and D. Zeppenfeld, Tau polarization measurements at LEP and SLC, Phys. Lett. B 235, 198 (1990).

[59] J. H. Kühn and A. Santamaria, Tau decays to pions, Z. Phys. C 48, 445 (1990).

[60] S. Jadach, J. H. Kühn, and Z. Was, TAUOLA: A library of Monte Carlo programs to simulate decays of polarized tau leptons, Comput. Phys. Commun. 64, 275 (1991).

[61] K. Hagiwara, T. Li, K. Mawatari, and J. Nakamura, TauDecay: A library to simulate polarized tau decays via FeynRules and MadGraph5, Eur. Phys. J. C 73, 2489 (2013).

[62] D. G. Dumm, P. Roig, A. Pich, and J. Portoles, $\tau \rightarrow \pi \pi \pi \nu_{\tau}$ decays and the $a_{1}(1260)$ off-shell width revisited, Phys. Lett. B 685, 158 (2010). 